\title{
Convex Hull indexed Gaussian Mixture Model (CH-GMM) for 3D Point Set Registration
}

\author{
Jingfan Fan, Jian Yang*, Danni Ai, Likun Xia, Yitian Zhao, Xing Gao, and Yongtian Wang \\ Beijing Engineering Research Center of Mixed Reality and Advanced Display, School of Optics and Electronics, Beijing Institute of Technology, Beijing 10081, \\ $\underline{\text { China }}$
}

\section{ARTICLE INFO}

Keywords:

Convex hull

Gaussian Mixture Model

Point Sets

Non-rigid Registration

\section{Introduction}

Pairwise point set registration is an essential component in many computer vision applications, including model retrieval, stereo matching, model mosaic, motion tracking and shape recognition [1]. Pairwise registration aims to estimate the best transformation that aligns two models based on the shapes of their overlapping components. Over the past several decades, numerous 3D registration methods have been proposed. The iterative closest point (ICP) method by Besl et al. [2] is the most popular due to its simplicity and speed. Given point correspondence, transformation is updated by solving a least square problem and given transformation, point correspondence is recovered based on the nearest neighbor relationship. But the one-to-one hard assignment assumption of the method causes the optimization not to be stable and the method can easily be trapped in local minima.

In a different manner, the Gaussian mixture model (GMM) [3-11] based method works by modeling point sets using probability distributions and registration is achieved by seeking the transformation so that the two point sets exhibit similar GMM responses. The GMM-based method has been proven to be effective for point set registration. However, for point sets with similar shape but different densities or noise levels, the GMM responses can differ considerably. Consequently, the GMM- based methods can be trapped in local minima during optimization.

To address the difficulties of the above methods, a novel convex hull indexed Gaussian mixture model ( $\mathrm{CH}-\mathrm{GMM})$ is proposed in this paper for non-rigid registration of 3D point sets. The CH-GMM model is constructed in the following way: First, local positional noise is smoothed and coplanar points are removed through a principal component analysis (PCA)-based resampling method [12]. Then, the convex hulls of both point sets are robustly extracted by using the quick-hull algorithm [13]. Finally, the CH-GMM response is calculated based on the Gaussian mixture function over the convex hull of a point set, where three conditions, proximity, area conservation and projection angle, are considered to improve performance of the method. As a result, the CH-GMM response will remain consistent for point sets with similar shapes but different densities. Based on the proposed CH-GMM model, rigid registration is achieved by seeking the rigid transformation parameters yielding the most similar $\mathrm{CH}-\mathrm{GMM}$ responses for the two point sets, and non-rigid registration is achieved by optimizing the thin-plate spline (TPS) interpolation parameters of the vertices of the convex hull.

The major contributions of the paper are summarized as follows: First, the proposed CH-GMM model can be seen as an

* Corresponding author. Tel.: +86-10-68912565-18; fax: +86-10-68912565-18; e-mail: jyang@bit.edu.cn 
improvement of GMM to make the GMM measurement consistent for point sets with similar shapes but different densities. Secondly, the proposed CH-GMM based registration method can effectively prevent the iterative optimization process from falling into local minima, thus improving the stability of the registration method. Finally, since the construction of CH-GMM only involves convex hull, the computational complexity can be significantly reduced compared with state-of-the-art methods.

\section{Related Works}

To improve registration accuracy, numerous studies have preferred using numerous features to construct the corresponding relationship of a point set; these features include the normal vectors of surfaces [14, 15], local invariant features [16, 17], curvatures [18-20], spin images [21], and textures [22-26]. Feature-based algorithms aim to establish a pairwise relationship (feature extraction) and to distinguish a limited number of invariable features from numerous points with respect to similarity transformation (matching). However, feature extraction and matching procedures are sensitive to imaging noise and resolution. Hence, a series of shape-based registration approaches has been proposed. The underlying shape representation [27, 28] is generally considered the most important component in registering arbitrary shapes. Deformable templates [29], Fourier descriptor [30], the snake model [31], and level set representation [32] have been proven to be powerful in estimating several local deformations; however, they require many parameters to represent shape deformation, which is time-consuming. Belongie et al. [33] introduced a shape context-based registration method that established correspondence by incorporating the neighborhood structure of point sets. However, this method is insufficiently robust for a wide variety of real objects. Zheng et al. [34] formulated the registration procedure as an optimization problem to preserve local neighboring structures. For this algorithm, each mesh point of a structure is interpreted as a node in a simple graph, and two neighboring nodes are connected by an edge. The matching of two graphs is then transformed to maximize the number of matched edges. This method utilizes many continuous optimization techniques for registration. However, this procedure may result in many outliers or considerable occlusion; that is, a significant degeneration of local structures may occur, which can lead to algorithm failure. Paragios et al. [35] suggested a signed distance transform for shape alignment that could be extended to any dimension. For this algorithm, signed distance transforms are utilized to constrain the optimization criterion to achieve global and local pixel-wise deformation estimation. Ma et al. [36] adopted $\mathrm{L}_{2}$ minimizing estimation to approximate the transformation from correspondences for non-rigid point set registration. This method can recover point correspondences iteratively and remove noise and outliers effectively. Chui et al. [37-39] proposed TPS robust point matching to estimate non-rigid transformations between point sets by using spatial mapping and outlier rejection to establish correspondences and to optimize warping parameters. Lian et al. [40, 41] suggested that the traditional robust point matching method could be reduced to a concave function by eliminating transformation variables and applying linear transformation. Concave optimization benefits correspondence problems and achieves a globally optimal solution without regularizing transformations. Deng et al. [8] cast the point cloud matching problem as a Schrodinger distance transform (SDT) representation problem. SDT is a measure of square root densities; it ensures the identification of shape distance with geodesic length and enables geodesic length to be expressed as a compact analytic expression on a unit Hilbert sphere. Aiger et al. [42] developed a four-point congruent set approach by extracting all coplanar four-point sets from source point sets that were approximately congruent with a given set of coplanar four points in the target point set under rigid transformation.

As mentioned earlier, most ICP or shape-based algorithms can be classified generally as hard assignment methods, which require the accurate calculation of corresponding pairs between different objects. However, solving the correspondence issue is difficult, particularly when point sets are affected by noise and nonlinear deformations. By contrast, the soft assignment technique has been proposed for fuzzy correspondence optimization; it allows for one-to-many relaxations or uncertain correspondences. Filtering methods are a means to realize soft assignment. Ma et al. [43, 44] proposed the use of a particle filter for rigid point set registration. The unscented Kalman filter (UKF) and Gaussian filters were utilized to determine the Gaussian approximation of the posterior for each particle. However, the algorithm requires numerous particles to perform accurate registration, which entails high computational costs for large data sets. Moghari et al. [45] introduced the UKF algorithm to register the rigid transformation of two point sets corrupted with additive Gaussian noise. The transformation parameters were reformulated as state vectors and estimated using UKF, which utilized the unscented transform and the true nonlinear model to approximate the distribution of the state random variable. The algorithm was evaluated based on point sets extracted from a pelvic cadaver through computed tomography (CT) and a scaphoid bone phantom through tracked free-hand ultrasound imaging. Sandhu et al. [46, 47] introduced particle filtering and stochastic dynamics for rigid point set registration. In their study, the registration process was constrained by the non-parametric prediction of filtering, and the uncertainty of transformation estimation was solved using dynamic schemes. This method requires no annealing schedule, and thus, it can reduce computational complexity and maintain the detailed information of point sets.

The probability density distribution approach is also a type of soft assignment technique. It assumes that each point of a source model corresponds to a weighted sum of the target points under a constraint of probability density distribution, instead of the closest target point alone; therefore, point set registration can be considered a means of aligning two density distributions by minimizing their discrepancies. Goldberger et al. [48] regarded the Kullback-Leibler divergence between two Gaussian mixtures of registered images, which was utilized to estimate the unscented transform between the images, as a similarity measure. On the basis of kernel correlation theory [3], the GMM proposed by Jian et al. $[4,5]$ demonstrated that point set registration could be reformulated as the static alignment of two Gaussian mixtures, and the best transformation could be obtained by minimizing the discrepancy measure between two corresponding mixtures. From this framework, they used distance to calculate rigid and nonrigid transformations between two point sets. However, GMM is known to be excessively sensitive when data points are few because of its high sensitivity to outliers. To address this problem, Gerogiannis et al. [11] proposed a student's t-mixture model (SMM), which originated from a wide class of elliptically symmetric distributions with the number of degrees of freedom. SMM obtains the corresponding partitioning of image pixels into clusters, and each cluster is represented by a corresponding density component. This model leads to a more robust outcome on outlying pixels. Wassermann et al. [7] presented an anatomical structure registration method with a GMM extended kernel mixture model by using tensor fields. This method computes a diffeomorphic transform between pairs of particle sets through a minimization process; it has the capacity to obtain 
an overlap rate higher than 70\%. GMM framework-based methods have been intensively studied in recent years because of their intuitiveness and simplicity. They generally require calculating the density distribution of each discrete point in the point set, and thus, time complexity is comparatively high for such methods. The weighting coefficients of all the points are equally distributed across the GMM framework. Hence, registration may fall into the local minimum for point sets with large density discrepancies or incomplete partials. Tao and Sun [9] proposed an asymmetrical GMM (AGMM) to match point sets. AGMM provides each Gaussian component with a different weight, which is based on the feature similarity between the data point and the model point. It facilitates data points to increase their chances of forming a correspondence. Through AGMM, the framework can converge faster and be more robust to noise. According to the GMM registration framework, coherent point drift (CPD) [6] forces the GMM centroid to move coherently as a group. The group preserves the topological structure of the point sets. To achieve rigid registration, the CPD algorithm reparametrizes the GMM centroid with rigid parameters. Nonrigid registration is obtained by regularizing the displacement field and then using variational calculus to realize optimal transformation. Ge et al. [49] introduced a GMM-based point set registration with global-local topology preservation method by gathering CPD constraint and local linear embedding. Compared with most existing approaches, this method is more flexible because it does not require assumptions and certain initial conditions, and thus, is able to handle complex non-rigid articulated transformation.

\section{Gaussian Mixture Model}

GMM is a probability model used to describe the continuous density functions of point sets, which have been widely used in many areas, including signal processing and image analysis. If $\mu_{i}, i=1,2, \ldots, n$ is assumed to represent the coordinates of each individual point in a point set, then GMM can be defined as follows:

$$
p(x)=\sum_{i=1}^{n} \phi\left(x \mid \mu_{i}, \sum_{i}\right)
$$

where $x$ represents the coordinate of a space point, $n$ is the number of points in the point set, and $\Sigma_{i}$ is the covariance matrix. Each individual point $\mu_{i}$ will generate a Gaussian function $\phi\left(x \mid \mu_{i}, \sum_{i}\right)$ at space point $x$ :

$$
\phi\left(x \mid \mu_{i}, \sum_{i}\right)=\frac{\exp \left[-\frac{1}{2}\left(x-\mu_{i}\right)^{T} \sum_{i}^{-1}\left(x-\mu_{i}\right)\right]}{\sqrt{(2 \pi)^{d}\left|\operatorname{det}\left(\sum_{i}\right)\right|}}
$$

where $d$ is the dimension of the point set. Hence, for point set registration, a GMM response $p(x)$ can be obtained for each point in space. Then the global GMM function of a point set is composed of the GMM responses for all the points in space. The general assumption states that if two point sets are identical, then they should have the same GMM responses [4]. For point set registration, GMM can be used to measure the distance between two point sets and the transformation between the two point sets can be recovered by minimizing this distance.

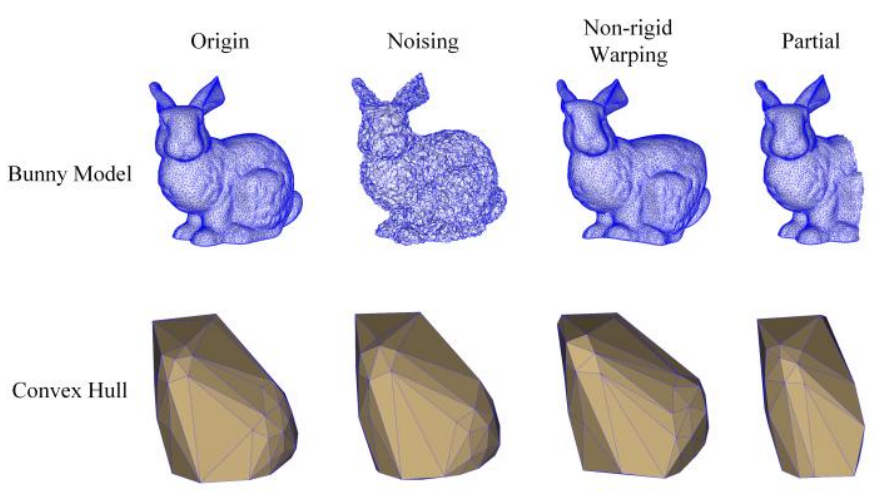

Fig. 1 The corresponding convex hull of the bunny model in different situations.

\section{Convex Hull Indexed Gaussian Mixture Model}

Based on the definition of GMM, GMM will be quite different for point sets with similar shapes but different density or GMM will change quite dramatically when point set undergoes local deformation. To overcome these limitations, this paper extends the GMM model to CH-GMM model by introducing the topological structure based weighting coefficients on the convex hull. The convex hull is initially extracted from the point set. Thereafter, the topological representation of a reference triangular facet is formulated by combining three conditions, namely, proximity, area conservation, and projection angle. The proximity makes $\mathrm{CH}-\mathrm{GMM}$ more robust to the local deformation and non-rigid matching problem; the area conservation guarantee the consistency of CH-GMM response to point sets with similar shapes but different density; the projection angle increase the sensitivity of CH-GMM to the geometrical structure. Finally, the conditions on the convex hull are utilized to achieve an approximate position. Then, the complete model, including the internal point feature, is considered for refinement.

\subsection{Convex Hull Extraction}

The convex hull is generally defined as the smallest convex set that contains the entire point set, which is typically utilized to represent the outline of an object. The topological structure of the point set or mesh can be depicted by a small set of vertices. If $M \in \mathfrak{R}^{3}$ is assumed as a point set in $3 \mathrm{D}$ space with $n$ points, then the vertex set of its convex hull can be defined as follows:

$$
C(M)=\{x \mid x \in M \& x \notin C(M-\{x\})\}
$$

where point $x$ in $M$ is considered the vertex of $C(M)$ if it satisfies the following condition: $x$ is a vertex of convex hull if removing it resulting in a different smaller convex hull.

The convex hull has been proven to be unique for a given point set, and it exhibits the property of affine invariance [50], which is suitable for registering point sets with affine transformations. However, due to the interference of noise and the existence of coplanar points, the triangulation of the convex hull may vary for the similar point sets. To eliminate the negative effects of noise and coplanar points, this paper introduces the point set resampling method [12], which regularizes the points and stabilizes the triangulation of the convex hull. This method initially performs the PCA algorithm to obtain the principal component of the point set. Thereafter, the method iteratively resamples the point set to achieve denoising and smoothing. Once the point set is regularized, the quickhull algorithm [13] is utilized for rapid convex hull extraction because of its small memory space requirement and fast processing speed. For this algorithm, if $n$ is considered the number of points in $M$, and $m$ 
is the number of vertices of $C(M)$, then computational complexity will be approximately $O(n \log m)$. The number of facets on the convex hull is generally significantly less than the number of points [50]; $m \leq 2 n-4$ is the upper bound in the limiting case. In the registration task, the 3D model is typically polluted by noise, non-rigid warping, and scraping. However, the structure of the convex hull remains stable as shown in Fig. 1.

\subsection{Weighting Coefficient Determination}

The proposed $\mathrm{CH}-\mathrm{GMM}$ defines a novel feature descriptor to represent the topological distribution of a point set in space, which extends GMM by introducing vertex and facet constraints into the convex hull. For each reference facet on the convex hull, three principles are introduced into GMM to preserve the topological structure of the entire point set. (1) The close facet relative to the reference facet has a larger contribution than farther facets. (2) The weighting triangular facets with the same area should equally contribute to the Gaussian mixture response of the reference facet. (3) The facet with less angle difference with respect to the reference facet should have a larger Gaussian mixture response. In referring to the principles, the weighting coefficients of the other facets, which are constructed based on three conditions, namely, proximity, area conservation, and projection angle, are designed to ensure that $\mathrm{CH}-\mathrm{GMM}$ will be robust to different density distributions and local deformations.

To guarantee that the Gaussian mixture contribution of the close facet relative to the reference facet is larger than that of farther facets, the Gaussian mixture contribution of the reference facet should be inversely proportional to the square distance of the centroid point of the reference and weighting facets. Such coefficient-designing method guarantees that the weighted Gaussian mixture can represent local deformation, which makes $\mathrm{CH}-\mathrm{GMM}$ more robust to the non-rigid matching problem.

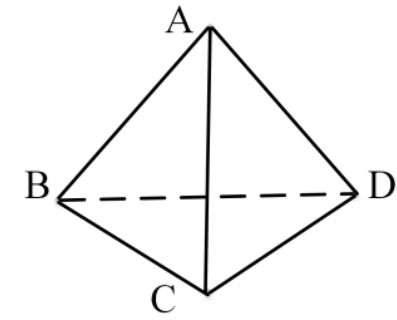

(a)

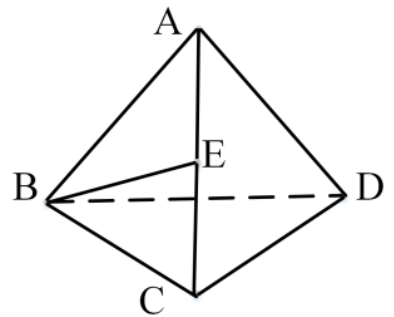

(b)
Fig. 2 The schematic diagram of different triangular decomposition.

The area conservation principle indicates that the weighting triangular facet with the same area should equally contribute to the Gaussian mixture response of the reference facet. Given that the convex hull extraction procedure may lead to different triangular decompositions of the same point set, the sensitivity of $\mathrm{CH}-\mathrm{GMM}$ responses to different decomposition results should be reduced. Therefore, the coefficient of the weighting facet is designed to be proportional to the size of its area in this paper. Fig. 2 shows an example of triangular decomposition with respect to the $\mathrm{CH}-\mathrm{GMM}$ value of the reference facet $\triangle A C D$. The figure highlights that although different triangulations exist for the weighting facet $\triangle A B C$ [in (a), $\triangle A B C$ is the weighting facet; otherwise, in (b), both $\triangle A B E$ and $\triangle B C E$ are the weighting facets], the facet $\triangle A B C$ in (a) should have an effect similar to the combined effects of $\triangle A B E$ and $\triangle B C E$ in (b). Hence, the triangular decomposition of the multiple acquired models of the same object may differ, but the area conservation principle can guarantee the consistency of $\mathrm{CH}-\mathrm{GMM}$ response.

The projection angle principle indicates that the Gaussian mixture contribution of the weighting facet is proportional to the projection length of the normal vector of the weighting facet to that of the reference facet. For this condition, the facet with less angle difference with respect to the reference facet should have the larger Gaussian mixture response. Such definition of the coefficient can considerably reduce Gaussian mixture response for facets with different normal directions.

Consider $F_{i}$ and $\vec{n}_{i}$ as the ith triangular facet and its corresponding normal vector on the convex hull, respectively; $s_{i}$ as the area of the $i^{\text {th }}$ triangular facet; $C P\left(F_{i}\right)$ as the centroid point of the triangular facet $F_{i}$; and $d_{i j}$ as the distance between the centroid points of the $i^{\text {th }}$ and $j^{\text {th }}$ triangular facets $F_{i}$ and $F_{j}$. On the basis of the aforementioned analysis, the weighting coefficient of the $j^{\text {th }}$ to the $i^{\text {th }}$ facet can be defined as follows:

$$
\omega_{i j}=\frac{\mathrm{s}_{j}\left(\vec{n}_{i} \cdot \vec{n}_{j}\right)}{\mathrm{d}_{i j}^{2}},\left(i \neq j, \mathrm{~d}_{i j} \neq 0\right)
$$

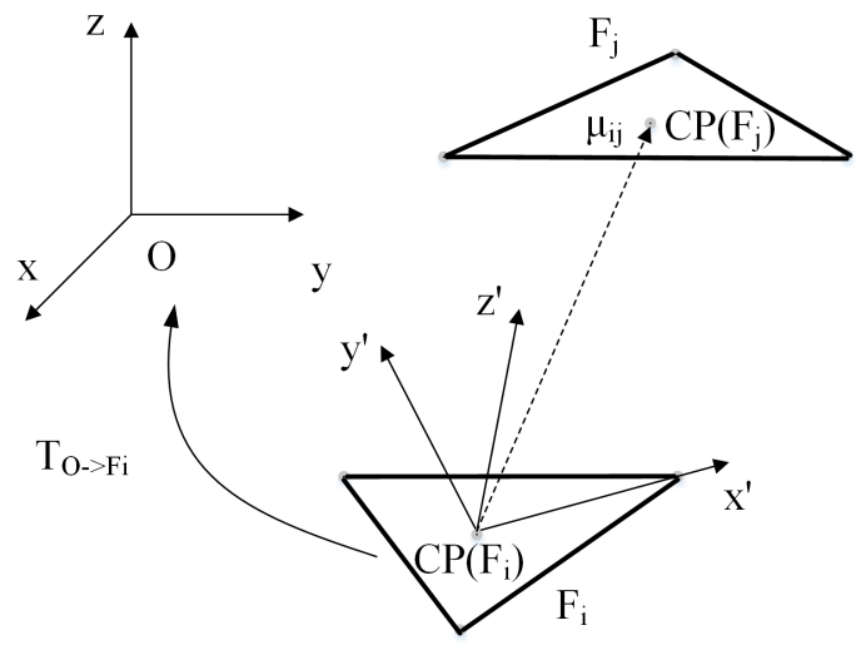

Fig. 3 The schematic diagram of the weighting coefficient.

Suppose that the reference facet $F_{i}$ has the coordinate system xyz, as shown in Fig. 3. For this coordinate system, the z-axis is defined by the normal vector of $F_{i}$, the x-axis is defined by the vector from centroid point $C P\left(F_{i}\right)$ to the farthest vertex of the triangle, and the $y$-axis is defined by the cross product of the $\mathrm{x}$ and z-axes. Therefore, the transformation matrix $T_{O->F_{i}}$ from the original coordinate system to the reference coordinate system can be determined. In addition, $\mathrm{CH}-\mathrm{GMM}$ can be reformulated as follows:

$$
\operatorname{CH}-\operatorname{GMM}\left(F_{i}\right)=\left\{\begin{array}{l|l}
p(x) & \begin{array}{l}
p(x)=\sum_{j=1}^{m} \omega_{i j} \phi\left(x \mid \mu_{i j}, \Sigma\right) ; \\
\mu_{i j}=C P\left(F_{j}\right) \cdot T_{O->F_{i}} ; \\
F_{i}, F_{j} \in \text { ConvexHull }(M) ; \\
x \in \mathfrak{R}^{3}
\end{array}
\end{array}\right\}
$$



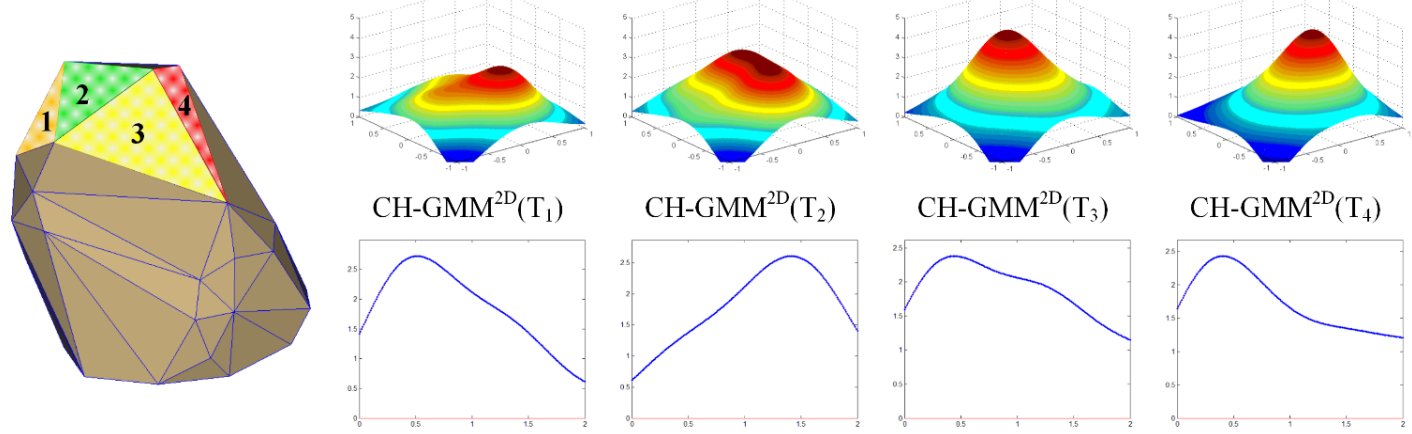

$\mathrm{CH}-\mathrm{GMM}^{2 \mathrm{D}}\left(\mathrm{T}_{4}\right)$
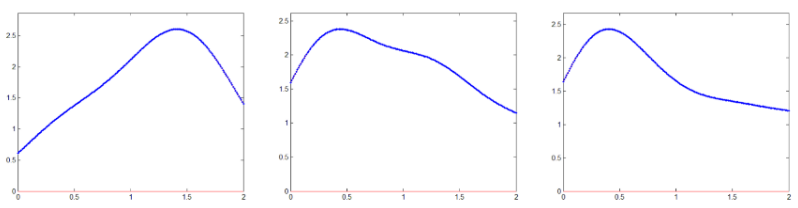

CH-GMM ${ }^{1 \mathrm{D}}\left(\mathrm{T}_{1}\right)$

$\mathrm{CH}-\mathrm{GMM}^{\mathrm{ID}}\left(\mathrm{T}_{2}\right)$

$\mathrm{CH}-\mathrm{GMM}^{\mathrm{ID}}\left(\mathrm{T}_{3}\right)$

$\mathrm{CH}-\mathrm{GMM}^{1 \mathrm{D}}\left(\mathrm{T}_{4}\right)$

Fig.4 Visualization of the CH-GMM responses for different reference facets. Left figure: convex hull and selected sampling facets. First row of right figure: 2D CH-GMM responses in the facet plane of the selected facets in left figure. Second row of the right figure: 1D CH-GMM responses in the normal direction of the facet plane of the selected facets in the left figure.

where $\mu_{i j}$ represents the relative position of $C P\left(F_{j}\right)$ in the reference coordinate system of $F_{i}$ by multifying transformation matrix $T_{O->F_{i}}$ with $C P\left(F_{j}\right) \cdot \mu_{i j}$ is invariant to the translation and rotation of the point cloud because $T_{O-F_{i}}$ indicates the position of the reference facet in the original coordinate system that corresponds to the global transformation of the model. Moreover, the weighed value $\omega_{i j}$ can be calculated using the relative position and angle, as illustrated in Equation (4). Therefore, $C H-G M M\left(F_{i}\right)$, which is calculated by $\mu_{i j}$ and $\omega_{i j}$, is invariant to the translation and rotation of the point cloud.

Fig. 4 illustrates four examples of the $\mathrm{CH}-\mathrm{GMM}$ response of different reference facets on the convex hull of a randomly generated point set. The left part shows the convex hull of a point set, in which the first to fourth facets are the selected four reference triangular facets. The first row of the right part corresponds to the 2D CH-GMM responses on the facet plane of the selected facets, whereas the second row of the right part shows the 1D CH-GMM responses in the normal direction of the facet plane of the selected facets. The first to fourth columns of the right figure correspond to the 2D and 1D CH-GMM responses of the selected facets in the left figure. The figure indicates that for different reference facets, the decomposed 1D and 2D responses exhibit different distributions, which represent the typical properties of the selected facet with respect to the other facets and points. For a determined point set in 3D space, the CH-GMM response of each facet is uniquely decided by its neighboring points and facets, which can then be utilized as a similarity measure for rigid and non-rigid registrations.

\section{Point Set Registration Algorithm}

Given that the CH-GMM responses of the similar point sets are proximate when they are arbitrary rigidly or limited nonrigidly deformed from a point set, the $L_{2}$ distance of CH-GMM responses can be utilized as a similarity measure for point set registration. This section introduces the target function of the optimization schemes for rigid and non-rigid registrations using the proposed CH-GMM.

\subsection{Rigid Registration}

The function $\mathrm{CH}-\mathrm{GMM}\left(F_{i}\right)$ is invariant to the translation and rotation of the point cloud because $\mathrm{CH}-\mathrm{GMM}\left(F_{i}\right)$ is composed by area, direction, and the relative position of the reference facet $F_{i}$ and other facets. Therefore, the $L_{2}$ distance of CH-GMM responses is utilized as a similarity measure for rigid registration.
Consider $F_{i}^{A}$ as the $i^{\text {th }}$ triangular facet of point set A and $F_{j}^{B}$ as the $j^{\text {th }}$ triangular facet of point set B. Then, the CH-GMM responses of $F_{i}^{A}$ and $F_{j}^{B}$ are denoted as $\mathrm{CH}-\mathrm{GMM}\left(F_{i}^{A}\right)$ and $\mathrm{CH}-\mathrm{GMM}\left(F_{j}^{B}\right)$, respectively. According to Equation (5), both $\mathrm{CH}-\mathrm{GMM}\left(F_{i}^{A}\right)$ and $\mathrm{CH}-\mathrm{GMM}\left(F_{j}^{B}\right)$ are continuous field functions, and their difference can be calculated using $L_{2}$ distance as follows:

$$
d_{L_{2}}\left(F_{i}^{A}, F_{j}^{B}\right)=\int\left[\mathrm{CH}-\mathrm{GMM}\left(F_{i}^{A}\right)-\mathrm{CH}-\mathrm{GMM}\left(F_{j}^{B}\right)\right]^{2} d x
$$

Furthermore, it can be decomposed as follows:

$$
\begin{gathered}
d_{L_{2}}\left(F_{i}^{A}, F_{j}^{B}\right)=\int\left[\mathrm{CH}-\operatorname{GMM}\left(F_{i}^{A}\right)-\mathrm{CH}-\operatorname{GMM}\left(F_{j}^{B}\right)\right]^{2} d x \\
=\int \mathrm{CH}-\operatorname{GMM}\left(F_{i}^{A}\right)^{2} d x+\int \mathrm{CH}-\operatorname{GMM}\left(F_{j}^{B}\right)^{2} d x \\
-2 \times \int \mathrm{CH}-\operatorname{GMM}\left(F_{i}^{A}\right) \times \mathrm{CH}-\operatorname{GMM}\left(F_{j}^{B}\right) d x
\end{gathered}
$$

where $\int \mathrm{CH}-\mathrm{GMM}\left(F_{i}^{A}\right)^{2} d x$ can be calculated as follows:

$$
\begin{aligned}
& \int \operatorname{CH}-\operatorname{GMM}\left(F_{i}^{A}\right)^{2} d x=\int \sum_{k=1}^{m_{A}} \omega_{i k}^{A} \phi\left(\mathrm{x} \mid \mu_{i k}^{A}, \Sigma_{A}\right) \times \sum_{l=1}^{m_{A}} \omega_{i l}^{A} \phi\left(\mathrm{x} \mid \mu_{i l}^{A}, \Sigma_{A}\right) d x \\
& =\sum_{k=1}^{m_{A}} \sum_{l=1}^{m_{A}} \omega_{i k}^{A} \omega_{i l}^{A} \int \phi\left(\mathrm{x} \mid \mu_{i k}^{A}, \Sigma_{A}\right) \times \phi\left(\mathrm{x} \mid \mu_{i l}^{A}, \Sigma_{A}\right) d x
\end{aligned}
$$

where $m_{A}$ and $m_{B}$ are the numbers of facets of the convex hull of point sets $A$ and $B$. Let

$$
\int \phi\left(\mathrm{x} \mid \mu_{1}, \Sigma_{1}\right) \phi\left(\mathrm{x} \mid \mu_{2}, \Sigma_{2}\right) d x=\phi\left(0 \mid \mu_{1}-\mu_{2}, \Sigma_{1}+\Sigma_{2}\right)
$$

Then, Equation (9) can be simplified as follows:

$$
\int \mathrm{CH}-\mathrm{GMM}\left(F_{i}^{A}\right)^{2} d x=\sum_{k=1}^{m_{A}} \sum_{l=1}^{m_{A}} \omega_{i k}^{A} \omega_{i l}^{A} \phi\left(0 \mid \mu_{i k}^{A}-\mu_{i l}^{A}, \Sigma_{A}+\Sigma_{A}\right) d x
$$

Similarly, Equation (7) can be further calculated as follows:

$$
\begin{gathered}
d_{L_{2}}\left(F_{i}^{A}, F_{j}^{B}\right)=\int\left[\operatorname{CH}-\operatorname{GMM}\left(F_{i}^{A}\right)-\operatorname{CH}-\operatorname{GMM}\left(F_{j}^{B}\right)\right]^{2} d x \\
=\sum_{k=1}^{m_{A}} \sum_{l=1}^{m_{A}} \omega_{i k}^{A} \omega_{i l}^{A} \phi\left(0 \mid \mu_{i k}^{A}-\mu_{i l}^{A}, \Sigma_{A}+\Sigma_{A}\right) \\
+\sum_{k=1}^{m_{B}} \sum_{l=1}^{m_{B}} \omega_{j k}^{B} \omega_{j l}^{B} \phi\left(0 \mid \mu_{i k}^{B}-\mu_{i l}^{B}, \Sigma_{B}+\Sigma_{B}\right) \\
-2 \times \sum_{k=1}^{m_{A}} \sum_{l=1}^{m_{B}} \omega_{i k}^{A} \omega_{j l}^{B} \phi\left(0 \mid \mu_{i k}^{A}-\mu_{i l}^{B}, \Sigma_{A}+\Sigma_{B}\right)
\end{gathered}
$$

The problem of point set registration can be reformulated as finding two similar triangular facet pairs $\left\{F_{i}^{A}, F_{j}^{B}\right\}$, for which $d_{L_{2}}$ is minimized. The following equation is then obtained: 


$$
\left\{F_{i}^{A}, F_{j}^{B}\right\}=\underset{i, j}{\operatorname{argmin}} d_{L_{2}}\left(F_{i}^{A}, F_{j}^{B}\right)
$$

As shown in Equation (12), the computation complexity of $d_{L_{2}}\left(F_{i}^{A}, F_{j}^{B}\right)$ is $O\left(m_{A} m_{B}\right)$. In order to achieve the minimizing target, each facet $F_{i}^{A}$ should only be compared with a few facets $F_{j}^{B}$, which have a similar shape, because a facet pair with different shapes leads to a different $\mathrm{CH}-\mathrm{GMM}$ response. Therefore, the computation complexity of the minimization process is $O\left(m_{A}^{2} m_{B}\right)$. The calculation burden for optimization is comparatively small, and the best matching facet pair can be easily determined because the number of triangular facets on the convex hull is significantly minimized compared with that of the entire point set.

When the matched triangular facet pair is obtained, rigid transformation between all the matched facets is calculated using the Umeyama's method [51]. Thereafter, the obtained transformation may be utilized to transform the entire point set and achieve global rigid transformation $T^{*}$ between the two point sets.

\section{A. Non-Rigid Registration}

On the basis of the aforementioned rigid registration process, non-rigid global transformation can be approximated by combining the CH-GMM framework with TPS transformation. Thus, the vertices of a convex hull are considered the control points, which exhibit the following merits. a) The vertices of the convex hull are already generated and regularly distributed around the entire point set. Therefore, the control points can effectively preserve the topological representation of the point set. b) The number of vertices on the convex hull is significantly less than that of the entire point set, which can considerably improve calculation efficiency. Compared with the vertices in the regular gridding method, the vertices on the convex hull are independent of the density distribution of the points, and thus, are more effective and robust in representing the local transformation of the entire point set.

Let all the vertices of the convex hull $\left\{c_{1}, c_{2}, \ldots, c_{n}\right\}$ be used as the control points of TPS transformation. Then, $n$ denotes the number of points, and $W$ represents the transformation fields of the control points; thus, the coordinate of the point transformed by TPS can be calculated using

$$
T P S(\mathrm{p})=\mathrm{W} \cdot D(\mathrm{p})+p
$$

where $D(\mathrm{p})$ represents the weighting coefficient that corresponds to an arbitrary point $p$ and can be calculated as follows:

$$
D(\mathrm{p})=\left[\begin{array}{c}
\mathrm{U}\left(\left|\mathrm{p}-\mathrm{c}_{1}\right|\right) \\
\mathrm{U}\left(\left|\mathrm{p}-\mathrm{c}_{2}\right|\right) \\
\ldots \\
\ldots \\
\mathrm{U}\left(\left|\mathrm{p}-\mathrm{c}_{n}\right|\right)
\end{array}\right]
$$

Moreover, the following equation is provided: $U\left(\left|\mathrm{p}-\mathrm{c}_{i}\right|\right)=-\left|\mathrm{p}-\mathrm{c}_{i}\right|$. Hence, the non-rigid registration of $\mathrm{CH}-$ GMM can be defined as follows:

$$
\underset{W}{\arg \min } d_{L_{2}}=\underset{W}{\arg \min } \int\left[C H-G M M_{T P S\left(P_{A}\right)}\left(F_{i}^{A}\right)-C H-G M M_{P_{B}}\left(F_{j}^{B}\right)\right]^{2} d x
$$

where $\mathrm{P}_{A}$ and $\mathrm{P}_{B}$ represent the control points of point sets $\mathrm{A}$ and $\mathrm{B}$, respectively. $\left\{F_{i}^{A}, F_{j}^{B}\right\}$ is the best matching triangle pair in Equation (12). The objective of the function is to minimize $d_{L_{2}}$ between the CH-GMM responses of the TPS-transformed $\mathrm{P}_{A}$ and that of the original $\mathrm{P}_{B}$, and Equation (15) can be decomposed in a similar manner as Equation (11). Hence, by nonlinearly optimizing the transformation field $\mathrm{W}$, the best non-rigid transformation of each control point can be achieved.

\section{B. Implementation Details}

The CH-GMM algorithm for the registration of point sets $A$ and $B$ can be summarized into the pseudocode shown in Table 1.

Table 1. Pseudocode for CH-GMM algorithm.

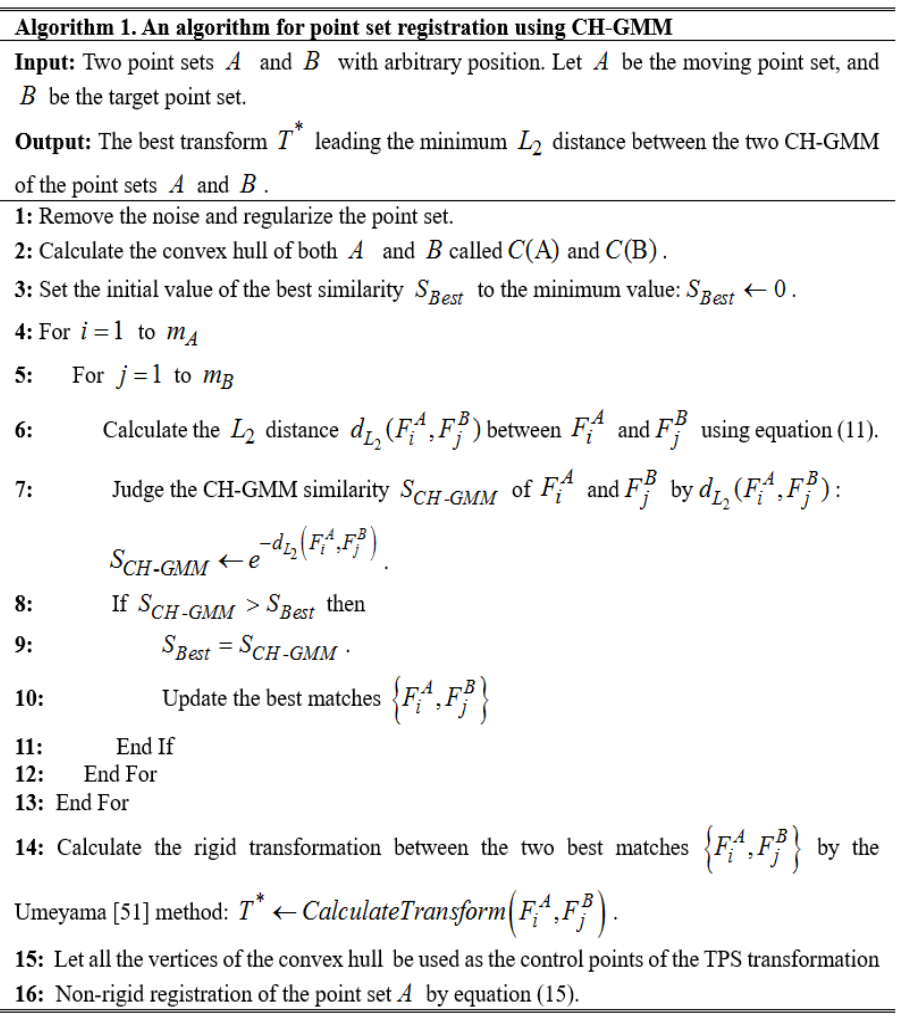

\section{Experimental Results}

In this paper, a series of experiments is designed to test the performance of the proposed and current algorithms with respect to noise interference, density difference, and partial overlapping. To facilitate the quantification of registration accuracy, the bounding boxes of all the data sets are normalized to scales of 0 to 100 . For each designed experiment, our algorithm is compared with the ICP [2], CPD [6], and GMM [4] algorithms, as mentioned in the first section. Registration accuracy is evaluated through the root-mean-square error, which is calculated as the sum of the Euclidean distances of each corresponding point pair between the source and the target point sets. All the algorithms are written in $\mathrm{C}++$ language, and the experiments are conducted using a standard PC with $16 \mathrm{~GB}$ RAM and $3.4 \mathrm{GHz}$ Intel CPU.

Fig. 5 shows the design used to evaluate the robustness of the proposed method with respect to rotational change between two point sets. Spider point data are obtained from the Aim@SHAPE Shape Repository [52]. In this figure, the red spider is the target point set, whereas the blue point sets are the source point sets 


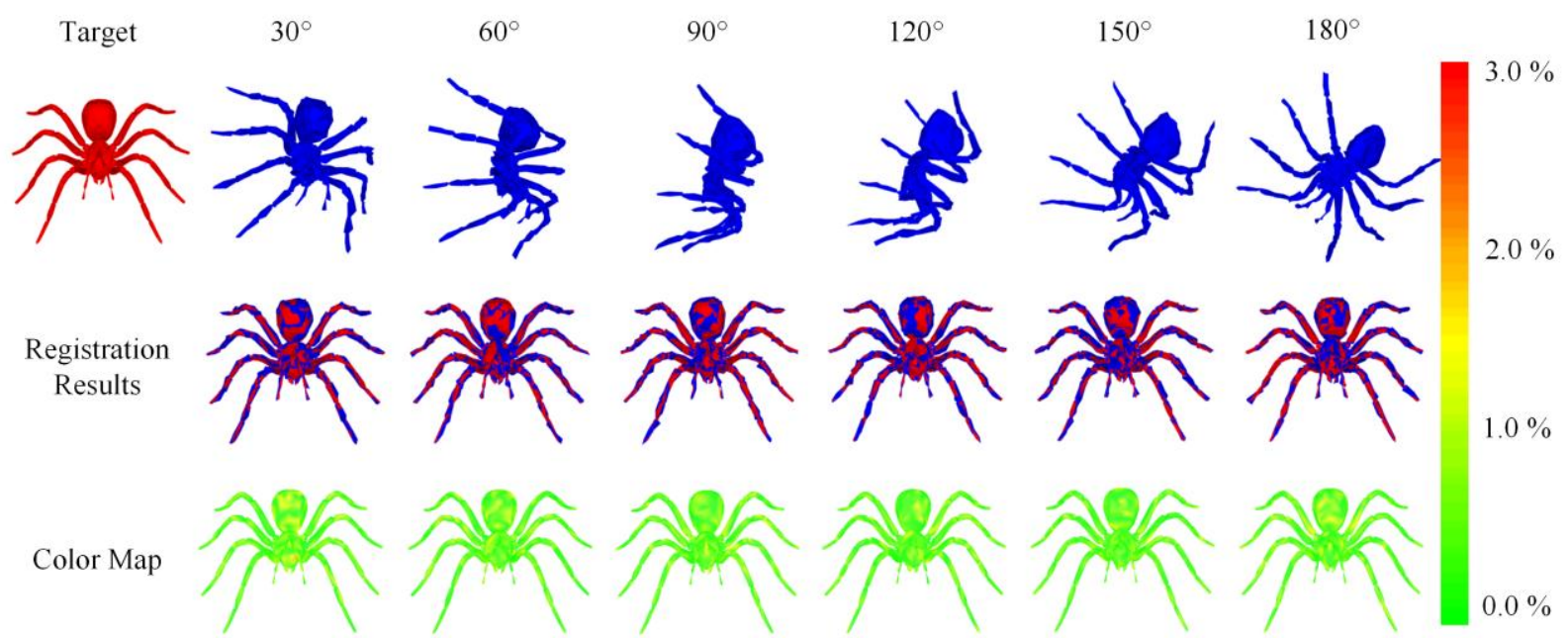

Fig. 5 Rigid registration results of point sets with different rotation angles. The first row: the target model (red) and source model (blue), which are generated from the target model with $30^{\circ}$ to $180^{\circ}$ rotation angles. The second row: registration results of the target and source models. The third row: the color map rendering of distance error be-tween the target and the registered models.

generated from the source point set, with a rotational change ranging from $30^{\circ}$ to $180^{\circ}$. Without sacrificing generality, the source point sets are randomly translated within a range of 100 300 , and the point sets are polluted by Gaussian noise with a standard deviation of $\sigma_{G}=1.0$. The second row shows the registration results of the target and source point sets, whereas the third row provides the color map rendering of the distance error between the target and the registered point sets. The registration results show that the two point sets are clearly perfectly registered together, and the area with an error greater than $1 \%$ is reasonably smaller relative to the entire figure.

Fig. 6 compares the performances of ICP, CPD, GMM, and $\mathrm{CH}-\mathrm{GMM}$ under different initial rotation angles. For the rotation angles of $30^{\circ}$ and $60^{\circ}$, all the four methods evidently perform well for the registration of the point sets. When the initial angle increases to $90^{\circ}$, the error of ICP is considerably larger than those of the other three methods, whereas the error of GMM remains as small as that of CH-GMM. When the initial angle increases from $120^{\circ}$ to $180^{\circ}$, the errors of ICP, CPD, and GMM are all considerably larger than that of CH-GMM. For the initial angle of $180^{\circ}$, the registration errors of ICP, CPD, GMM, and CHGMM are $7.25 \%, 5.92 \%, 6.31 \%$, and $0.74 \%$, respectively. Thus, the proposed CH-GMM algorithm is evidently robust under different initial rotation angles. It outperforms the other three algorithms, particularly with large rotation angles.

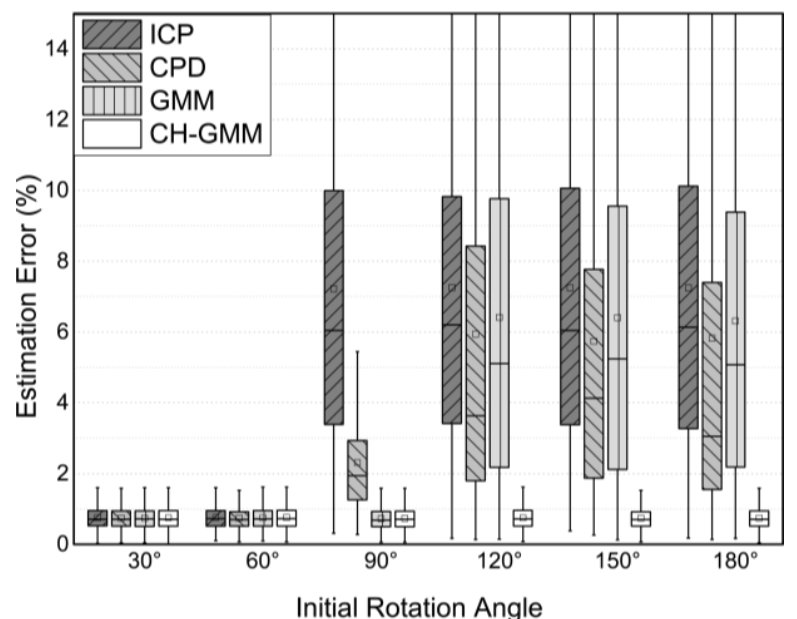

Fig. 6 Comparison of different algorithms for rigid registration over different rotation angles.

Fig. 7 illustrates the performance of the CH-GMM algorithm for point sets with different noise and outlier scales. The bear data set is obtained from the Aim@SHAPE Shape Repository [52]. The red image shows the original model, whereas the
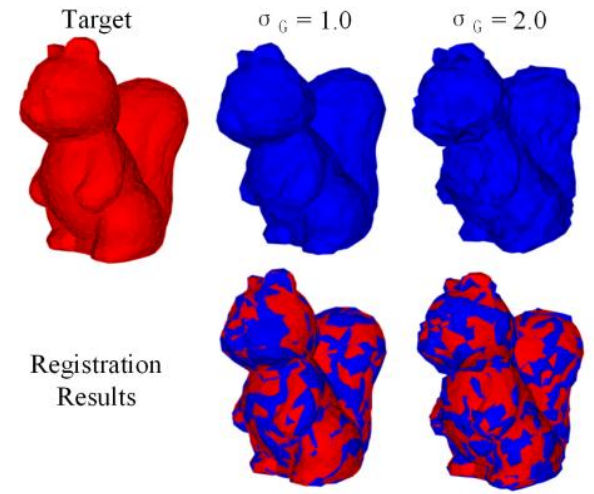

Registration Results

Color Map
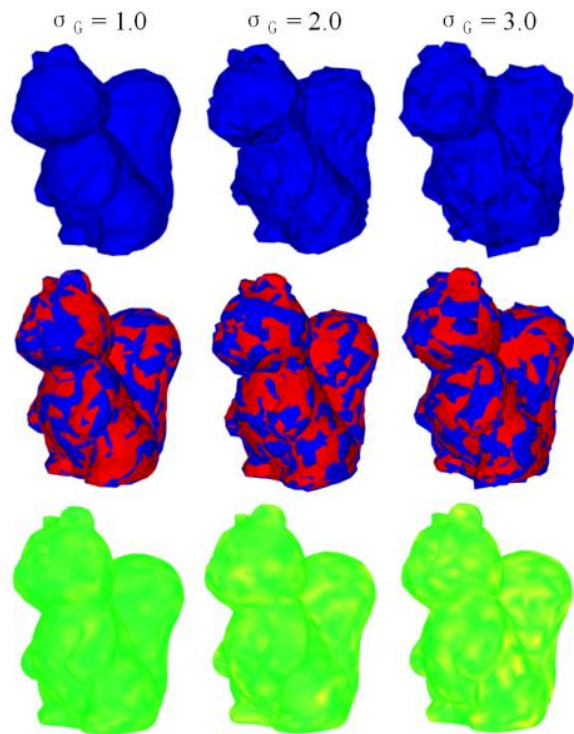
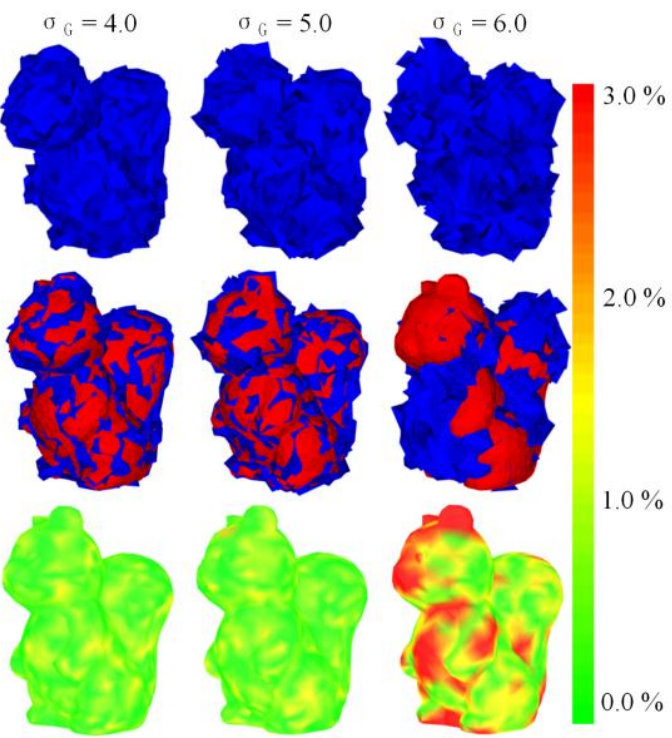

Fig. 7 Rigid registration of point sets with different scale of Gaussian noises. First row: target model (red) and the source models (blue), the source models are generated from the target model with a different scale of Gaussian noise. The second row: registration results of the target and source models. Third row: the color map rendering of distance error between the target and the registered models. 
succeeding images are generated by randomly translating every vertex in the model in three spaces, wherein the translations for each vertex in the three axes are independent and the amount of translations is within the controlled Gaussian distribution with a standard deviation ranging from $\sigma_{G}=1.0$ to $\sigma_{G}=6.0$. Similarly, the source point sets are randomly translated within the range of $100-300$ and are randomly rotated within the range of $30^{\circ}-60^{\circ}$. The second row of the figure shows the fusion rendering of the original and registered models, whereas the third row shows the color map rendering of the registration errors in the entire model. According to this figure, registration error evidently increases as noise level increases. However, the registration results are still highly accurate, and deviations are negligible for a standard deviation of less than 6.0. Although the registration errors of $\sigma_{G}=6.0$ are apparently large in some local parts of the model, the source and target models remain globally aligned.

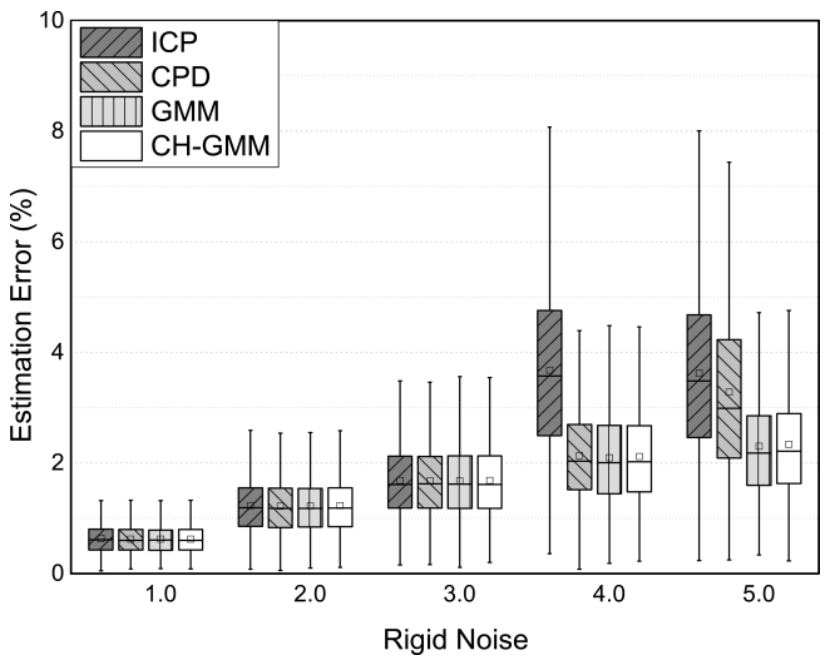

Fig. 8 Comparisons of different algorithms for rigid registration over varying scale of Gaussian noises.

Fig. 8 compares the performance of CH-GMM with those of the ICP, CPD, and GMM algorithms in the presence of noise and outliers. The figure shows that registration errors increase as Gaussian noise increases for all the compared methods. However, ICP fails to register the models when Gaussian noise is larger than 4.0, whereas CPD obtains a large noise of $3.63 \%$ when Gaussian noise reaches 5.0. Moreover, the estimated mean errors at $\sigma_{G}=5.0$ for the GMM and CH-GMM algorithms are $2.31 \%$ and $2.34 \%$, respectively, which show that both algorithms perform effectively and consistently under all Gaussian noise levels.

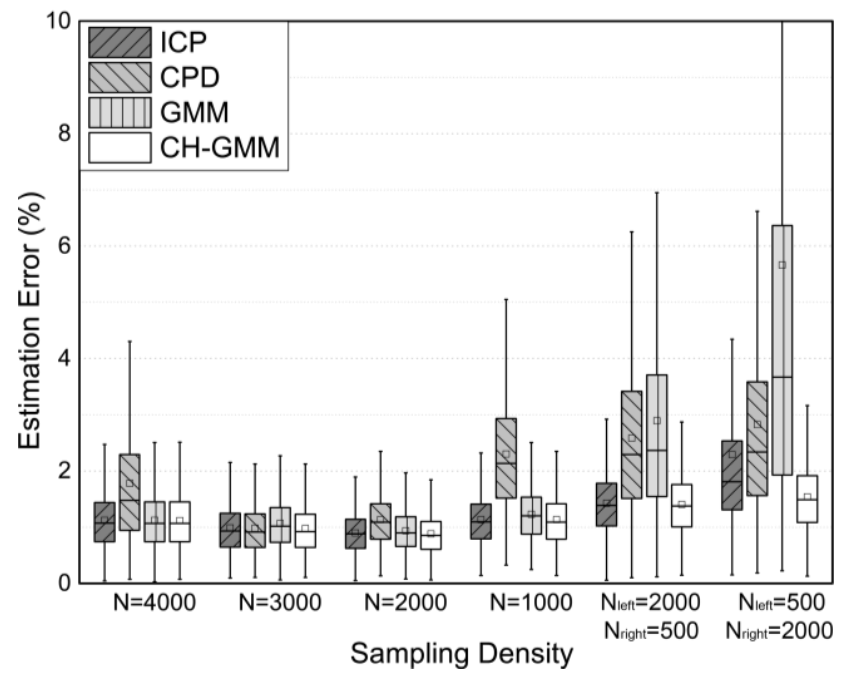

Fig. 10 Comparisons of different algorithms for rigid registration over varying sampling densities.

Fig. 9 shows the registration results of different sampling densities. The rabbit data are obtained from the Stanford 3D Scanning Repository [53]. The red image shows the original model, whereas the succeeding blue images are generated by choosing a specific percentage of the points from the entire point set and applying random translation, rotation, and scaling within a predefined range. The second row of the figure shows the fusion rendering of the original and registered models, whereas the third row shows the registration errors in the entire model. This figure illustrates that although sampling densities are different under various sampling ratios, the proposed $\mathrm{CH}-\mathrm{GMM}$ algorithm can register the two models accurately. Evidently, the average errors for all the sampling ratios are distributed within the range of $0 \%-1.5 \%$. The largest registration errors are approximately $3.0 \%$; these errors are locally distributed at the bottom right sections of the rabbit data and account for only a small ratio of the entire point set. However, the data errors are reasonably minimal relative to the entire rabbit.
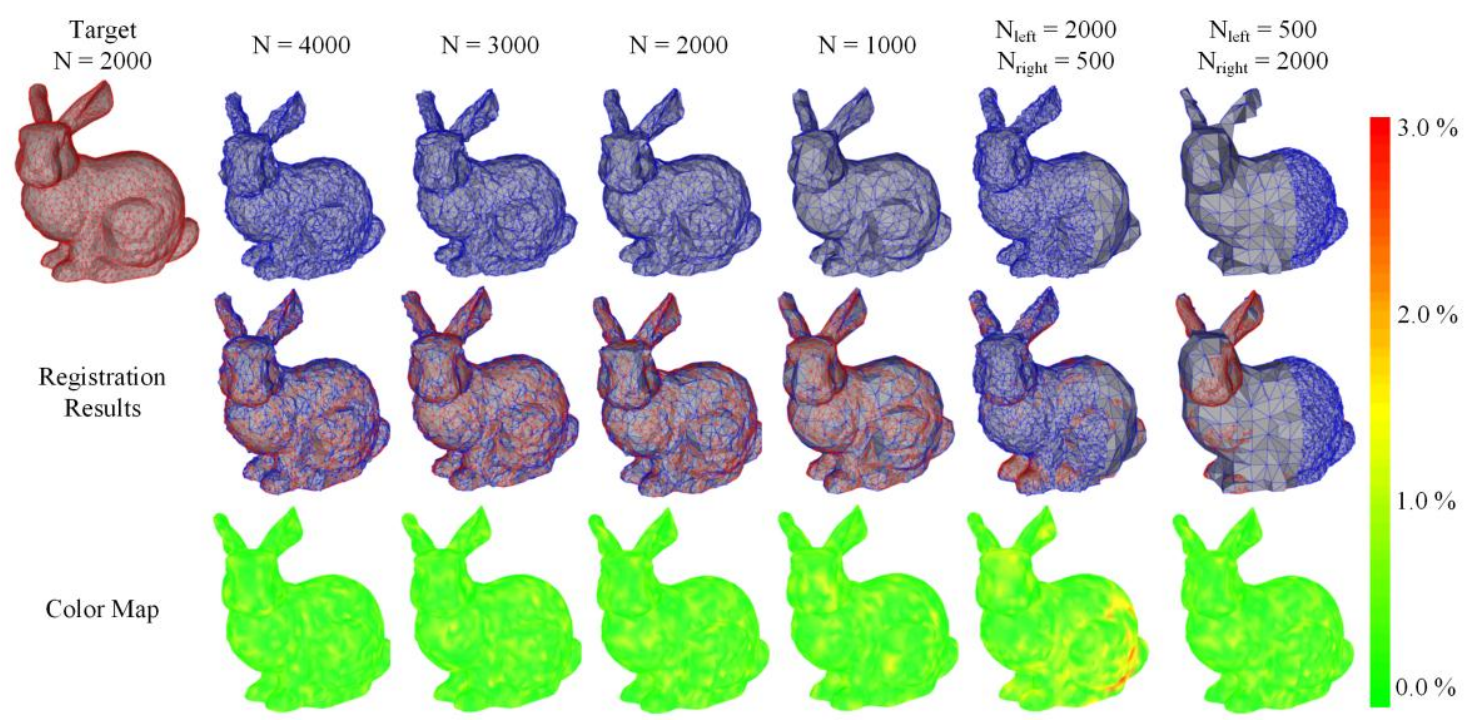

Fig. 9 Rigid registration of point sets with different sampling densities. Top row: target (red) and source (blue) models with varying sampling density ratios that correspond to the target model. The second row: registration results of the target and source models. The third row: the color map rendering of distance error between the target and the registered models. 

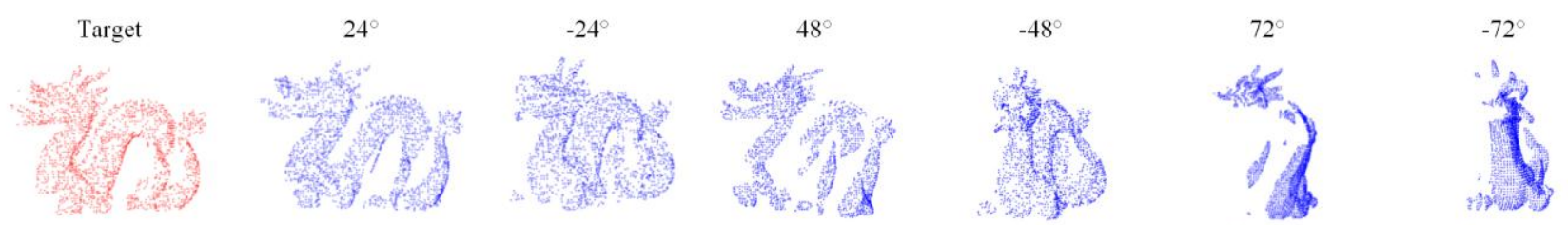

Registration Results
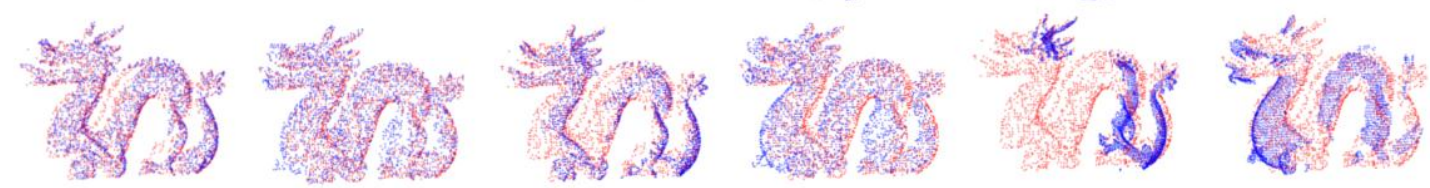

Fig. 11 Part-to-whole rigid registration of point sets. The first row: target model (red) and source model (blue) with different 3D data sampling angles corresponding to the target model. The second row: registration results of the target and source models.

Fig. 10 presents the estimated error of $\mathrm{CH}-\mathrm{GMM}$ compared with those of ICP, CPD, and GMM against sampling density. The figure shows that the errors estimated for the equal sampling density of the left $N_{\text {left }}$ and the right $N_{\text {right }}$ are approximately identical among all the compared methods. However, the errors estimated for ICP, CPD, and GMM severely increase with different sampling densities of the left and right parts. The mean errors estimated with a sampling density of $\mathrm{N}=2,000$ for ICP, CPD, GMM, and CH-GMM are $0.90 \%, 1.46 \%, 0.94 \%$, and $0.88 \%$, respectively. For the data set with sampling densities of $N_{\text {left }}=500$ and $N_{\text {left }}=2000$, the maximum errors recorded are over $4.48 \%$ for ICP, over $6.75 \%$ for CPD, over $10 \%$ for GMM, and less than $3 \%$ for $\mathrm{CH}-\mathrm{GMM}$, with mean errors of $2.29 \%$, $2.83 \%, 5.66 \%$, and $1.54 \%$, respectively. The evaluation results show that the proposed CH-GMM method performs the best among all the methods in various sampling density cases, which only slightly affect the proposed method.

Fig. 11 shows the performance of CH-GMM for the part-towhole rigid registration problem. The dragon data sets are obtained from the Stanford 3D Scanning Repository [53]. The red image shows the target point set, whereas the blue point sets are generated from the 3D data sampling with scanning angles of $\pm 24^{\circ}, \pm 48^{\circ}$, and $\pm 72^{\circ}$. The second row of the figure shows the registered results of the source point to the point sets that correspond to the first row. The overlapping areas are clearly shown in this figure, which indicates that the CH-GMM algorithm performs well in all the rotation angles. In general, even for the imaging angle of $\pm 72^{\circ}$, in which only a few overlapping point sets exist in the head and tail of the dragon, the proposed method can still obtain the best registration results.

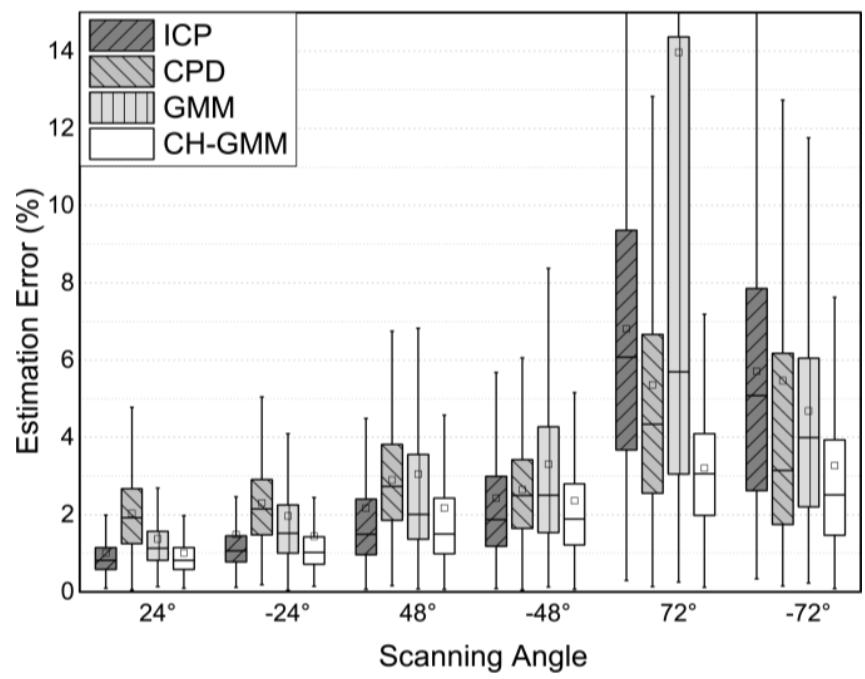

Fig. 12 Comparisons of different algorithms for rigid registration over varying 3D data scanning angles.

Fig. 12 illustrates the performances of CH-GMM, ICP, CPD, and GMM in terms of robustness to different scanning angles. The figure shows that registration errors also increase as the sampling angles increase in all the compared methods. Although ICP appears effective and can obtain the lowest error similar to that of $\mathrm{CH}-\mathrm{GMM}$ with smaller angles of $\pm 24^{\circ}$ and $\pm 48^{\circ}$, ICP

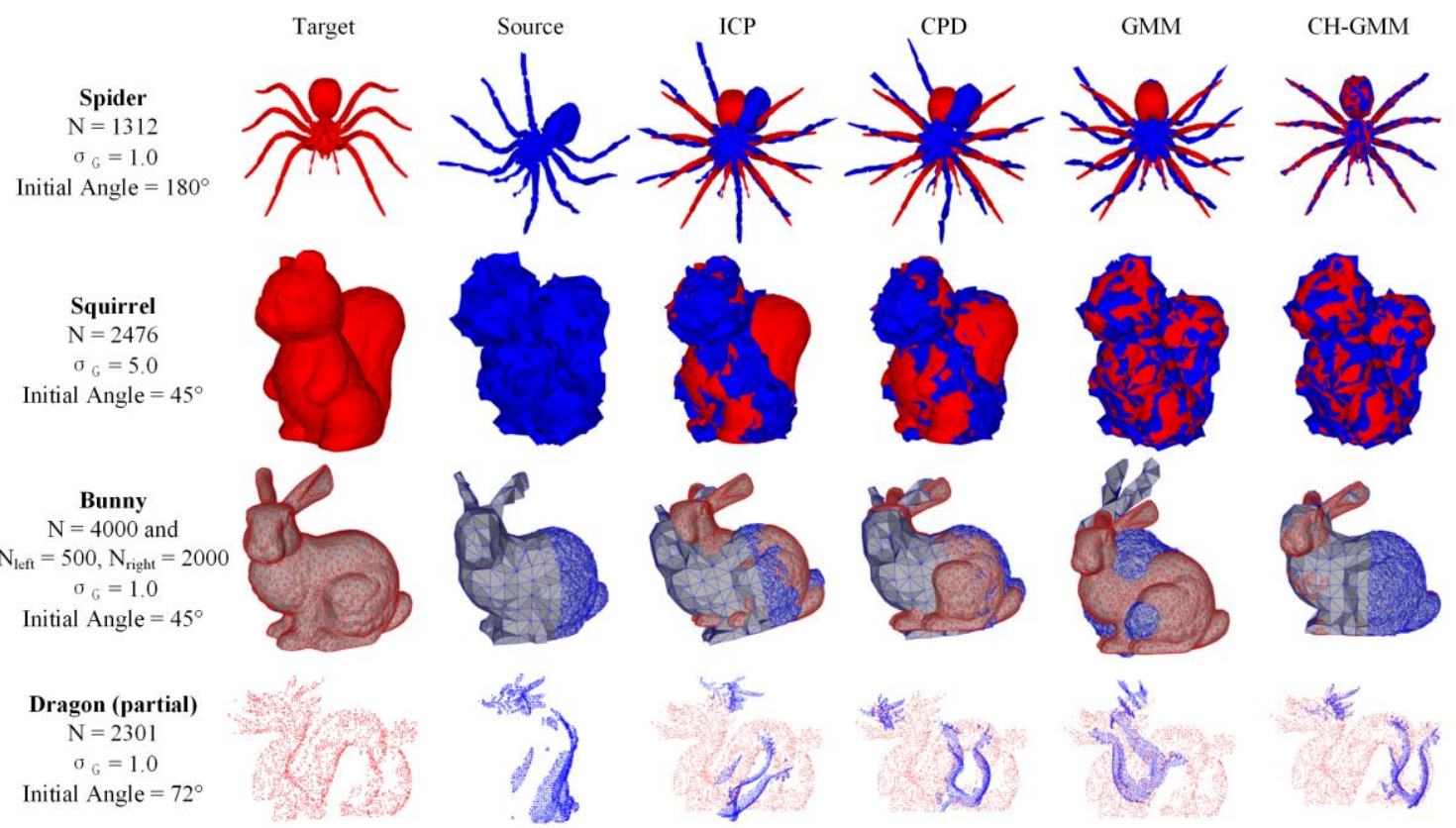

Fig. 13 Comparisons of different algorithms for rigid registration for multiple models. 


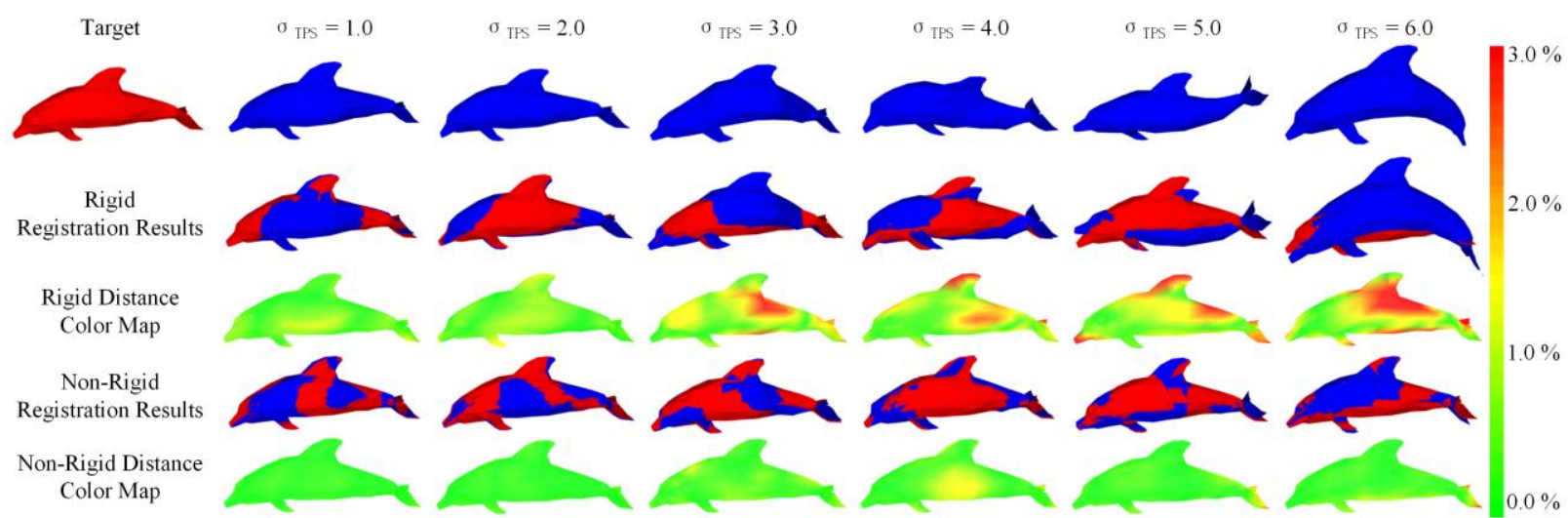

Fig. 14 Rigid and non-rigid registrations of dolphin point sets. Top row: target model (red) and source model (blue) with non-rigid deformation scales. The second and fourth rows: rigid and non-rigid registration results of the target and source models, respectively. The third and fifth rows: the color map rendering of distance error between the target and the rigid and non-rigid registered models. fails to register the models when the sampling angles reach $\pm 72^{\circ}$. This failure can be attributed to the fact that the overlap ratios of the point sets at the angles of $\pm 24^{\circ}$ and $\pm 48^{\circ}$ are considerably larger than those at the angle of $\pm 72^{\circ}$. Therefore, except for our proposed method, all the other methods fail to register the models at the angle of $\pm 72^{\circ}$. The differences in the estimated errors of $\mathrm{CH}-\mathrm{GMM}$ under various angles are minimal compared with those of the other methods.

Fig. 13 shows the non-rigid registration results of various data sets using the ICP, CPD, GMM, and CH-GMM methods. The red data in the first column are the target point sets, whereas the blue data in the second column are the source point sets generated from the corresponding target point sets, with designated parameters tagged on the left of the source point set. For the spider model, the ICP and CPD methods fail to register the models with a large rotation angle. Although an overall registration is implemented using the GMM method, the details, such as the legs of the spider, are unregistered. By comparison, the CH-GMM method provides excellent registration results in both the overall and specific details of the spider. For the squirrel, ICP and CPD fail to register the models because of the considerable variation among them. By contrast, GMM and $\mathrm{CH}-$ GMM are well aligned. For the rabbit, different sampling and densities cause ICP to fail. CPD and GMM also fail to register the ears of the rabbit although its other parts have been registered. The proposed $\mathrm{CH}-\mathrm{GMM}$ registers the two models with an accurate registration result. For the dragon, several overlapping point sets cause ICP, CPD, and GMM to fail. However, this situation does not affect the effectiveness of the proposed CH-GMM method, which still achieves an accurate registration result. On the basis of the aforementioned results, the proposed method outperforms the other three methods in terms of the non-rigid registration of the model or point set.

Fig. 14 evaluates the performance of the proposed method with respect to the rigid and non-rigid registration results. The red dolphin obtained from the Aim@SHAPE Shape Repository [52] is set as the target, whereas the blue source data sets are generated from the target with different scales of the non-rigid Gaussian noise $\sigma_{T P S}$. Non-rigid noise is added to the control points of TPS interpolation, in which the translations for each control point in the three axes are independent, and the amount of translations is under the controlled Gaussian distribution with a standard deviation $\sigma_{T P S}$ ranging from 1.0 to 6.0 and an increment of 1.0. The rigid registration results of the target and source point sets are shown in the second row, whereas the color map rendering of the distance error that corresponds to the rigid registration results are shown in the third row. The rigid

registration error obviously increases as the level of $\sigma_{T P S}$ increases, particularly in a local location, such as the fin of the dolphin. The fourth row shows the non-rigid registration results of the source and target point sets, whereas the fifth row shows the color map rendering of the distance error that corresponds to the non-rigid registration results. These results clearly show that non-rigid registration errors remain reasonably small as the level of non-rigid deformation increases, whereas large errors are distributed in some specific local areas of the model (tail). Evidently, the non-rigid registration procedure is highly effective for the registration of the models, in which the non-rigid deformation of the model can be effectively identified and corrected. Notably, for a large non-rigid deformation with $\sigma_{T P S}=6.0$, the tail of the dolphin is directed downward in contrast with the upward direction in the target model. Nevertheless, CM-GMM remains capable of registering the two models together, and the mean registration error is approximately $1.01 \%$. In conclusion, the proposed method is highly effective for the registration of models or point sets with large-scale non-rigid deformation.

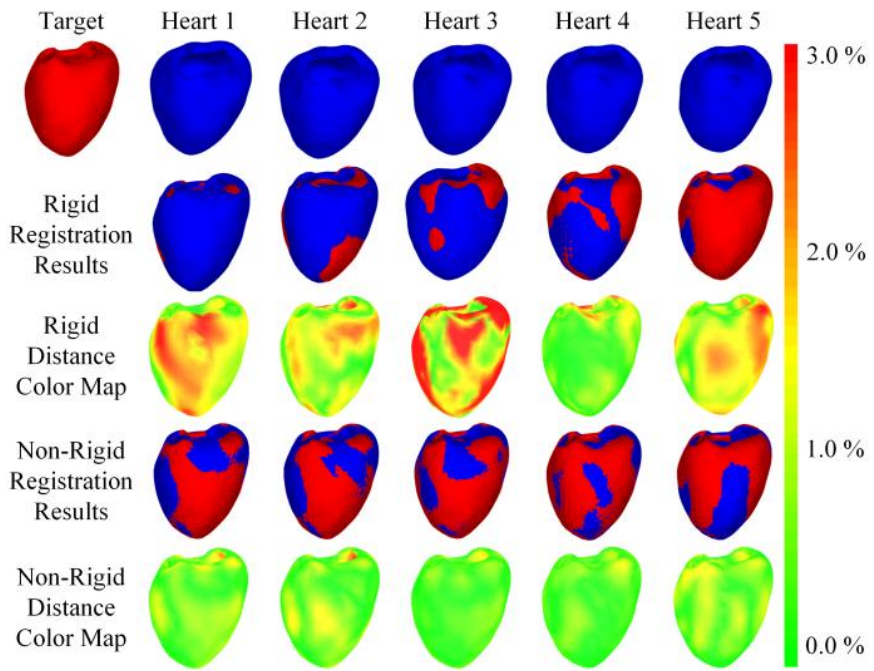

Fig. 15 Rigid and non-rigid registrations of heart point sets with different cardiac cycles. Top row: target model (red) in the cardiac phase of diastole and source model (blue) in the other six cardiac phase of contraction. The second and fourth rows: rigid and non-rigid registration results of the target and source models, respectively. The third and fifth rows: the color map rendering of distance error between the target and the rigid and non-rigid registered models, respectively.

Fig. 15 shows the rigid and non-rigid registration results of the heart models in six different cardiac phases. The heart data set is obtained from the Biomed Town Heart Model Data Set [54]. In 


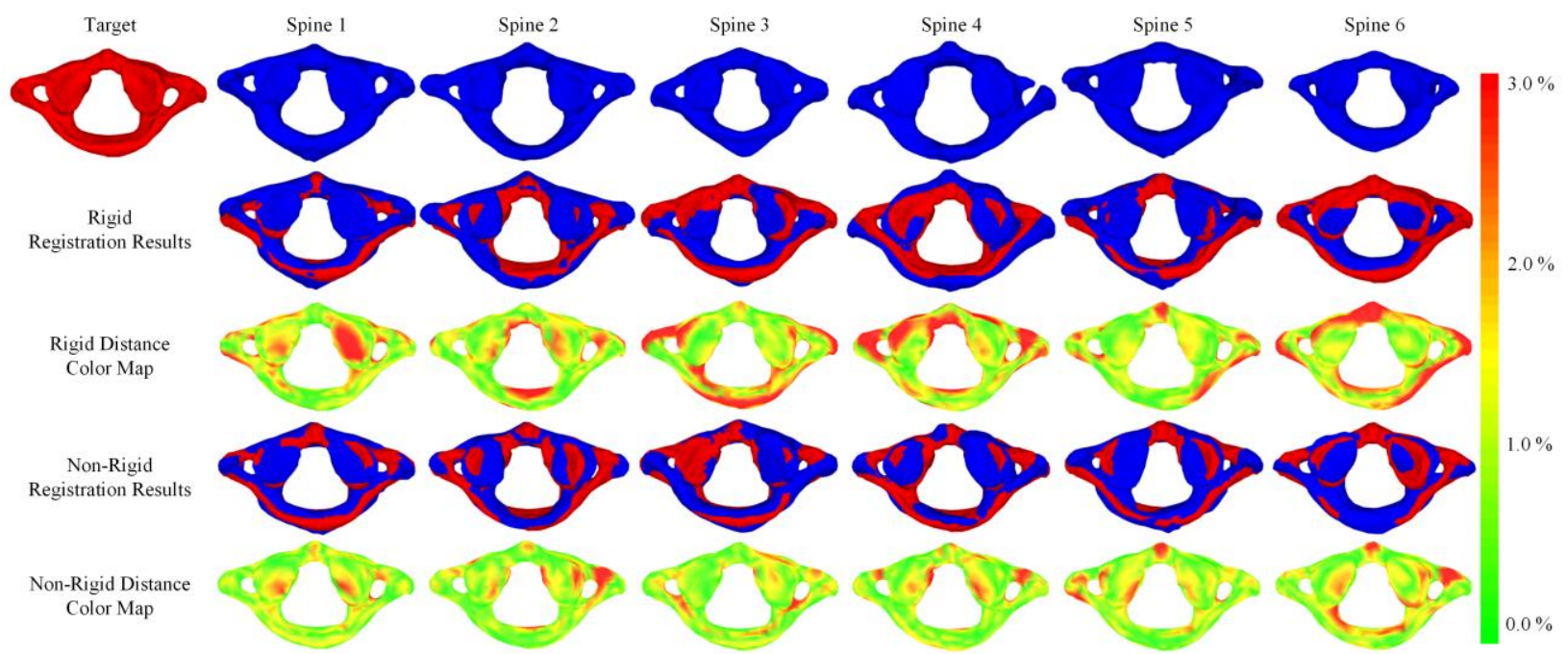

Fig. 16 Rigid and non-rigid registrations of spine point sets with different people. Top row: target model (red) and source models (blue). The second and fourth rows: rigid and non-rigid registration results of the target and source models, respectively. The third and fifth rows: the color map rendering of distance error between the target and the rigid and non-rigid registered models, respectively.

Fig. 15, the red heart model is the target model obtained in the cardiac phase of diastole, whereas the blue heart models are the source model obtained from the different cardiac contraction phases. The second row provides the rigid registration results of the target and source models, whereas the third row presents the color map rendering of the distance error that corresponds to the rigid registration results. Moreover, the non-rigid registration results and the corresponding color map rendering of the distance error are displayed in the fourth and fifth rows, respectively. The figure shows that with the contraction of the heart, the deformation between the two models increases. Evidently, the registration results with non-rigid registrations are significantly better than those with rigid registrations. Unlike the large error obtained by rigid registrations in global areas, non-rigid registrations can efficiently suppress distance error and deliver considerably precise registration results. Thus, significant applications of the proposed $\mathrm{CH}-\mathrm{GMM}$ include quantifying cardiac deformation and estimating the left ventricular ejection fraction.

Fig. 16 presents the rigid and non-rigid registration results of the spine models using CH-GMM. The spine data sets of seven people are obtained from the Southern Medical University, Guangzhou, China, in which the surface of the spine is manually segmented from CT volume data by a physician. The red spine model is the target data, whereas the succeeding blue models are the source data. The second and third rows in this figure show the rigid registration results and the corresponding color map rendering of the registration error, respectively. Meanwhile, the fourth and fifth rows show the non-rigid registration results and the corresponding color map rendering of the registration error, respectively. Compared with the rigid registrations with an average error rate of $2.15 \%$, the non-rigid registrations create two data sets that are reasonably registered together. Concurrently, the areas that suffer from the errors obtained from non-rigid registrations are considerably smaller than those obtained from the entire spine.

Fig. 17 shows the non-rigid registration results of CPD, GMM, and CH-GMM. Given that the ICP method is only used for rigid registration, this method is not included in this comparison. The non-rigid registration results and the corresponding color map rendering of the registration error are presented to solve different models, namely, the dolphin, the heart, and the spine. To illustrate the results clearly, the zoomed local parts are also shown in this figure. The first two rows display the registration results of the dolphin. The zoomed local parts indicate that a perfect non-rigid registration result is obtained using the CH-GMM method. In particular, approximately $98 \%$ of the points have registration errors less than $1 \%$ for the proposed method. The middle two rows present the registration results of the heart with different cardiac cycles, and the last two rows show the registration results of the spine from different people. The figure indicates that the CH-GMM method can produce the best alignment compared with the other two non-rigid registration methods.

Table 2. Comparison of non-rigid registration accuracy of varying methods over different data sets.

\begin{tabular}{cccccc}
\hline \hline \multicolumn{1}{c}{ Model } & Rigid Error & CPD & GMM & CH-GMM \\
\hline \multirow{6}{*}{ Dolphin } & $\sigma_{\mathrm{TPS}}=1.0$ & $1.25 \%$ & $1.32 \%$ & $0.27 \%$ & $0.29 \%$ \\
& $\sigma_{\mathrm{TPS}}=2.0$ & $1.29 \%$ & $1.33 \%$ & $0.34 \%$ & $0.44 \%$ \\
& $\sigma_{\mathrm{TPS}}=3.0$ & $2.67 \%$ & $2.71 \%$ & $0.83 \%$ & $0.96 \%$ \\
& $\sigma_{\mathrm{TPS}}=4.0$ & $2.73 \%$ & $2.76 \%$ & $1.01 \%$ & $1.03 \%$ \\
& $\sigma_{\mathrm{TPS}}=5.0$ & $3.02 \%$ & $2.79 \%$ & $1.07 \%$ & $0.93 \%$ \\
& $\sigma_{\mathrm{TPS}}=6.0$ & $4.33 \%$ & $3.37 \%$ & $1.17 \%$ & $1.01 \%$ \\
\hline \multirow{6}{*}{ Heart } & Heart 1 & $2.38 \%$ & $2.67 \%$ & $2.67 \%$ & $1.93 \%$ \\
& Heart 2 & $2.54 \%$ & $2.63 \%$ & $2.72 \%$ & $1.97 \%$ \\
& Heart 3 & $2.43 \%$ & $2.64 \%$ & $2.65 \%$ & $1.51 \%$ \\
& Heart 4 & $2.33 \%$ & $2.63 \%$ & $2.65 \%$ & $1.49 \%$ \\
& Heart 5 & $2.78 \%$ & $2.91 \%$ & $2.75 \%$ & $1.97 \%$ \\
& Heart 6 & $3.01 \%$ & $2.76 \%$ & $2.88 \%$ & $2.47 \%$ \\
\hline \multirow{6}{*}{ Spine } & Spine 1 & $2.06 \%$ & $3.52 \%$ & $1.87 \%$ & $1.64 \%$ \\
& Spine 2 & $2.09 \%$ & $3.56 \%$ & $1.76 \%$ & $1.76 \%$ \\
& Spine 3 & $2.24 \%$ & $3.50 \%$ & $1.95 \%$ & $1.74 \%$ \\
& Spine 4 & $2.43 \%$ & $3.62 \%$ & $1.94 \%$ & $1.83 \%$ \\
& Spine 5 & $2.18 \%$ & $3.50 \%$ & $2.25 \%$ & $1.81 \%$ \\
& Spine 6 & $2.15 \%$ & $3.39 \%$ & $2.08 \%$ & $1.66 \%$ \\
\hline \hline
\end{tabular}




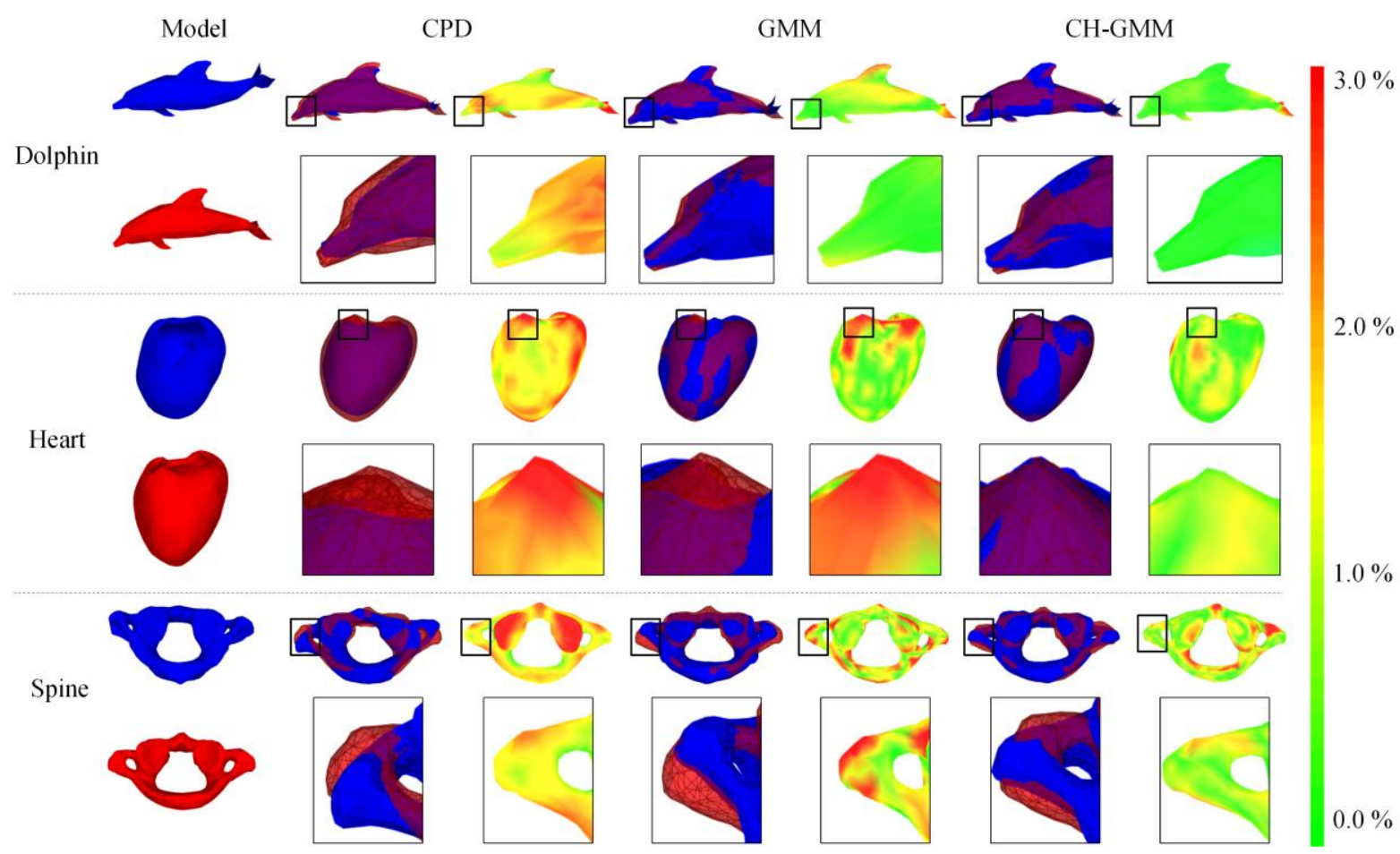

Fig. 17 Comparisons of different algorithms for non-rigid registration with multiple models in the original size and zoomed local parts.

For a quantitative comparison, the registration results of two different registration methods are reported for all the aforementioned models (dolphin, heart, and spine). The registration error from a pair of models is quantified as the average Euclidean distance. In particular, the rigid errors are illustrated for reference. As shown in Table 2, the non-rigid registration error of $\mathrm{CPD}$ is worse than that of its rigid registration error for most of the models. Although the CH-GMM method has a few parts with large registration errors, these errors can be ignored relative to those of GMM for a small-scale $\sigma_{T P S}$. Evidently, the proposed $\mathrm{CH}-\mathrm{GMM}$ can achieve the best registration results for all the tested models.

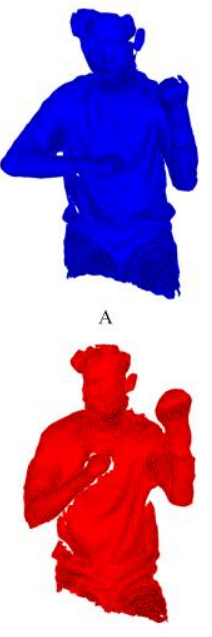

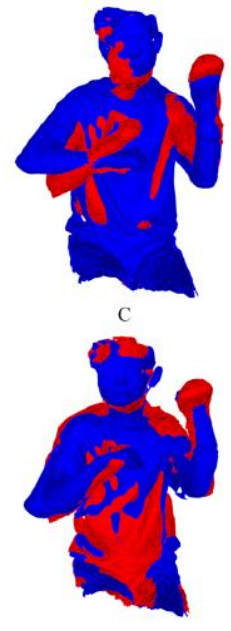

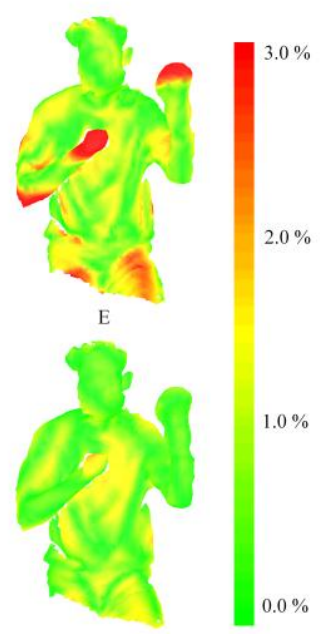

Fig.18 Experiments on models scanned by the Kinect device. A: Source model. B: Target model. C: Rigid registration results. D: non-rigid registration results. E: Distance map of the rigid registration errors. F: Distance map of the non-rigid registration errors.

Fig. 18 shows the upper parts of a human body scanned using the Kinect ${ }^{\mathrm{TM}}$ device. Fig. 18(A) shows the scanned data of a person with a horizontal right arm, whereas Fig. 18(B) shows the same person with a beveled right arm. The difference between the two scanning data sets can be observed clearly. Fig. 18(C) shows the fused results after the rigid registration of the two models. Fig. 18(E) presents the distance map of the registration error. This figure shows that the two models are roughly registered together, except for large errors found in the head and left arm. Statistically, the mean and largest errors of rigid registration are $1.57 \%$ and 5\%, respectively. Fig. 18(D) and Fig. $18(\mathrm{~F})$ show the combined results of the non-rigid registration results and the error distribution, respectively. These figures show that the two models are accurately registered and that less registration error can be identified. Statistically, the mean and largest errors are $0.78 \%$ and $1.97 \%$, respectively. Evidently, the proposed CH-GMM method is highly effective for the registration of models with global and local differences.

A comparison of computational times is presented in Table 3. The first and second columns show all the models and the corresponding number of points used in our experiments, respectively. The rigid registration methods (ICP, CPD, GMM, and $\mathrm{CH}-\mathrm{GMM}$ ) and the non-rigid registration methods (CPD, GMM, and CH-GMM) are investigated. Considering the rigid registration methods for the dolphin, the computational times of ICP, CPD, and GMM are nearly 3, 12, and 2 times, respectively, that of the proposed $\mathrm{CH}-\mathrm{GMM}$. With regard to the non-rigid registration methods for the rabbit, the computational time of GMM is nearly 8 times that of $\mathrm{CH}-\mathrm{GMM}$. The most impressive achievement shows that with a large number of points for a model, the computational time of CPD is extremely long, whereas that of $\mathrm{CH}-\mathrm{GMM}$ is acceptable. In conclusion, the proposed method has high time efficiency for the rigid and nonrigid registrations of the point sets or the models.

The optimum matching relationship between the triangles in different models is obtained by calculating the CH-GMM response of a triangle on the surface of the model convex hull. Geometric feature matching between the model surfaces is achieved and applied to model registration and target recognition. The elephant models with nine different poses (blue) and the standard elephant model (red) [55] are used to evaluate the feature matching and experimental results, as shown in Fig. 19. An excessive number of features are extracted to observe the matching results. Thus, a set of representative feature matching 
Table 3. Comparison of the time efficiency for rigid and non-rigid registrations with varying registration algorithms.

\begin{tabular}{ccccccccc}
\hline \hline \multirow{2}{*}{ Model } & \multirow{2}{*}{ Capicity } & \multicolumn{4}{c}{ Rigid(ms) } & \multicolumn{3}{c}{ Non-Rigid(ms) } \\
\cline { 3 - 9 } & & $I C P$ & $C P D$ & $G M M$ & $C H-G M M$ & $C P D$ & $G M M$ & $C H-G M M$ \\
\hline Dolphin & 897 & 197 & 879 & 145 & 76 & 1354 & 379 & 284 \\
Spider & 1312 & 852 & 1066 & 271 & 81 & 58685 & 861 & 751 \\
Dragon & 2301 & 2689 & 4013 & 997 & 146 & 137541 & 24323 & 3108 \\
Squirrel & 2476 & 3448 & 3971 & 1540 & 137 & 129737 & 24946 & 3479 \\
Spine & 3179 & 4017 & 23483 & 5798 & 293 & 197846 & 43845 & 7508 \\
Heart & 3481 & 4251 & 24587 & 6520 & 341 & 213479 & 47236 & 7876 \\
Bunny & 8176 & 13714 & 57614 & 11014 & 541 & $\infty$ & 119624 & 15327 \\
\hline \hline
\end{tabular}

results is presented, including the tips of two tusks and the center of the head, which are connected with green lines. The three features clearly accurately match (Elephant01-07 and 09), and a large deformation is produced when the elephant is in motion. Meanwhile, in the group Elephant08, the trunk and the tusks sharply curve downward and are located between the legs. The features of the tips of the two tusks are no longer on the surface of the convex hull. Therefore, error matching is produced because no matching point exists in the area of the tusks. Obviously, if the topology of the convex hull is maintained, then the proposed method guarantees the accuracy of the matching features, and a large non-rigid deformation is produced. By contrast, the correct matching features in the corresponding area cannot be obtained using the proposed method if the structure of the convex hull is changed.

Furthermore, CH-GMM is compared with three classic matching methods, namely, spin image [21], 3DSC [22], and SHOT [26]. The quantitative results of feature matching is shown in Table 4, where "Matches" represents the number of matching feature points, and "Inlier ratio" indicates the percentage of the correct matching feature points. "Inlier ratio" is calculated as follows. (1) The object model is deformed into the source model using the non-rigid registration method. (2) Relative distance error is calculated for the matching feature points with non-rigid registration. (3) Correct matching is obtained if the distance error is less than $2 \%$. Table 4 clearly shows that the average numbers of feature points for each method are 68.1, 54.3, 97.9, and 81.7, respectively, which satisfy the requirements of point cloud registration and target recognition. The average accuracies of spin image, 3DSC, and SHOT are relatively low because of the considerable non-rigid deformation $(37.6 \%, 49.7 \%$, and $58.5 \%$, respectively). Meanwhile, the average accuracy of $\mathrm{CH}-\mathrm{GMM}$ achieves $81.1 \%$ with respect to the best "Inlier ratio" of $92.5 \%$, which is significantly better than those of the other three methods. However, the matching accuracy of CH-GMM in the group Elephant08 is only $55.5 \%$ and is the worst result among all the groups because the topological structure of the convex hull is changed by non-rigid deformation, and correct matching cannot be achieved. Meanwhile, a matching accuracy of 55.5\% indicates that correct matching feature points are still obtained in the convex hull without structural modification.

\section{Discussion And Conclusions}

This paper proposes a novel CH-GMM method for the nonrigid registration of a point set or a model in $3 \mathrm{D}$ space. In general, GMM measures the probabilistic distribution of the point set in 3D space, which is sensitive to the difference in density and local deformation of the point sets. In addition, the convex hull is the tightest convex combination that contains all the points, which is unique for any point set and exhibits the properties of an affine invariant. Thereafter, three conditions of the convex hull, namely, proximity, area conservation, and projection angle, are weighted and combined with the GMM response. Therefore, the topological constraint of the entire point set is enhanced for GMM measures to adjust density variation and local deformation. In addition, the convex hull has significantly fewer points than the entire point set. Hence, the combination of GMM and the convex hull can accelerate the

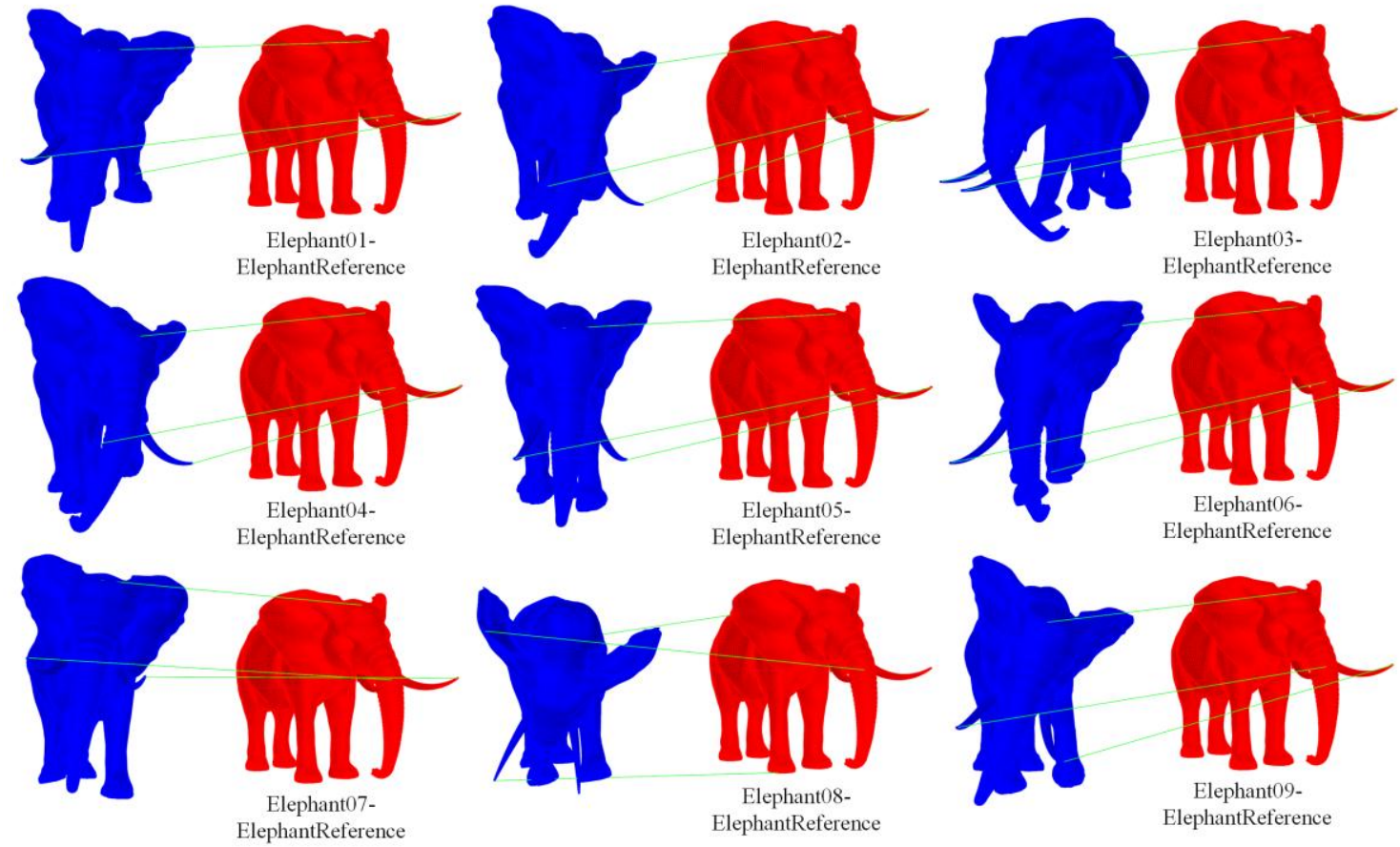

Fig.19 Feature matching experiments on elephant models in different poses. 
Table 4. Comparison of the feature matching results with varying recognition algorithms.

\begin{tabular}{c|cccccccc}
\hline \hline & \multicolumn{3}{|c}{ Spin image } & \multicolumn{2}{c}{$3 D S C$} & \multicolumn{2}{c}{ SHOT } & \multicolumn{2}{c}{ CH-GMM } \\
\cline { 2 - 9 } & Matches & Inlier ratio & Matches & Inlier ratio & Matches & Inlier ratio & Matches & Inlier ratio \\
\hline Elephant01 & 67 & $37.3 \%$ & 59 & $40.7 \%$ & 100 & $71.0 \%$ & 84 & $88.1 \%$ \\
Elephant02 & 71 & $46.5 \%$ & 59 & $52.5 \%$ & 102 & $52.9 \%$ & 90 & $84.4 \%$ \\
Elephant03 & 58 & $32.8 \%$ & 36 & $61.1 \%$ & 89 & $49.4 \%$ & 81 & $79.0 \%$ \\
Elephant04 & 76 & $36.8 \%$ & 67 & $46.3 \%$ & 114 & $68.4 \%$ & 84 & $83.3 \%$ \\
Elephant05 & 61 & $32.8 \%$ & 50 & $58.0 \%$ & 86 & $51.2 \%$ & 78 & $85.9 \%$ \\
Elephant06 & 68 & $41.2 \%$ & 38 & $36.8 \%$ & 100 & $57.0 \%$ & 93 & $92.5 \%$ \\
Elephant07 & 74 & $36.5 \%$ & 59 & $57.6 \%$ & 85 & $43.5 \%$ & 87 & $78.2 \%$ \\
Elephant08 & 64 & $34.4 \%$ & 60 & $43.3 \%$ & 112 & $63.4 \%$ & 63 & $55.5 \%$ \\
Elephant09 & 74 & $40.5 \%$ & 61 & $50.8 \%$ & 93 & $69.9 \%$ & 75 & $82.7 \%$ \\
Average & 68.1 & $37.6 \%$ & 54.3 & $49.7 \%$ & 97.9 & $58.5 \%$ & 81.7 & $81.1 \%$ \\
\hline \hline
\end{tabular}

registration process. Moreover, we utilize the vertices of the convex hull as the control points of TPS interpolation to achieve accurate global non-rigid registration.

To evaluate the performance of CH-GMM, a series of experiments is designed on simulated models, which are based on various aspects, including rotation angle, noise, density, part-topart registration, and various non-rigid deformation scales. According to the quantification results, the proposed method evidently outperforms the widely used ICP, CPD, and GMM with respect to the rigid and non-rigid registrations of point sets or models. Notably, CH-GMM is not only highly accurate in the registration of different noise scales and densities but is also highly efficient. Compared with ICP, CPD, and GMM, the computational efficiency of $\mathrm{CH}-\mathrm{GMM}$ is improved several times. Moreover, $\mathrm{CH}-\mathrm{GMM}$ is evaluated on different real medical data sets. The heart model in different cardiac cycles and the spinal bone models prove the effectiveness and robustness of the proposed method.

One limitation of the proposed method is that the candidate facet pairs on the convex hull should have similar shapes and $\mathrm{CH}-\mathrm{GMM}$ responses. Hence, on the one hand, if many outliers or noise are located outside the point sets, then the shapes of the convex hull of the source and target points will vary significantly and will lead to registration failure. On the other hand, if the models are both centrosymmetric point sets, then the timeconsuming characteristic of the registration procedure will be raised because the algorithm will find it difficult to search for correct pair candidates. Future research will focus on addressing the degeneration problem and GPU-based acceleration.

\section{Acknowledgments}

This work was supported by the National Basic Research Program of China (2013CB328806), the Key Projects in the National Science \& Technology Pillar Program (2013BAI01B01), the National Hi-Tech Research and Development Program (2015AA043203), the National Science Foundation Program of China (81430039, 61501030, 61572076).

\section{References}

[1] S. Ding, L. Lin, G. Wang, and H. Chao, "Deep feature learning with relative distance comparison for person re-identification," Pattern Recognition, vol. 48, pp. 2993-3003, 2015.

[2] P. J. Besl and N. D. McKay, "A method for registration of 3-D shapes," IEEE Trans. Pattern Analysis and Machine Intelligence, vol. 14, pp. 239-256, 1992.
[3] Y. Tsin and T. Kanade, "A correlation-based approach to robust point set registration," Computer Vision-ECCV 2004, pp. 558-569, 2004.

[4] B. Jian and B. C. Vemuri, "Robust Point Set Registration Using Gaussian Mixture Models," IEEE Trans. Pattern Analysis and Machine Intelligence, vol. 33, pp. 1633-1645, Aug 2011.

[5] B. Jian and B. C. Vemuri, "A robust algorithm for point set registration using mixture of Gaussians," Proc. Computer Vision-ICCV 2005, IEEE, 2005, pp. 1246-1251.

[6] A. Myronenko and X. Song, "Point Set Registration: Coherent Point Drift," IEEE Trans. Pattern Analysis and Machine Intelligence, vol. 32, pp. 2262-2275, 2010.

[7] D. Wassermann, J. Ross, G. Washko, W. M. Wells, and R. San Jose-Estepar, "Deformable Registration of Feature-Endowed Point Sets Based on Tensor Fields," Proc. Computer Vision and Pattern Recognition (CVPR), 2014 IEEE Conference on, 2014, pp. 2729-2735.

[8] Y. Deng, A. Rangarajan, S. Eisenschenk, and B. C. Vemuri, "A Riemannian Framework for Matching Point Clouds Represented by the Schrodinger Distance Transform," Proc. Computer Vision and Pattern Recognition (CVPR), 2014 IEEE Conference on, 2014, pp. 3756-3761.

[9] W. Tao and K. Sun, "Asymmetrical Gauss Mixture Models for Point Sets Matching," Proc. Computer Vision and Pattern Recognition (CVPR), 2014 IEEE Conference on, 2014, pp. 1598-1605.

[10] D. Breitenreicher and C. Schnörr, "Robust 3D object registration without explicit correspondence using geometric integration," Machine Vision and Applications, vol. 21, pp. 601-611, 2010.

[11] D. Gerogiannis, C. Nikou, and A. Likas, "The mixtures of Student's t-distributions as a robust framework for rigid registration," Image \& Vision Computing, vol. 27, pp. 12851294,2009

[12] H. Huang, S. H. Wu, M. L. Gong, D. Cohen-Or, U. Ascher, and H. Zhang, "Edge-Aware Point Set Resampling," Acm Transactions on Graphics, vol. 32, p. 12, Jan 2013.

[13] C. B. Barber, D. P. Dobkin, and H. Huhdanpaa, "The quickhull algorithm for convex hulls," ACM Transactions on Mathematical Software (TOMS), vol. 22, pp. 469-483, 1996.

[14] J. Feldmar and N. Ayache, "Rigid, affine and locally affine registration of free-form surfaces," International journal of computer vision, vol. 18, pp. 99-119, 1996.

[15] C. Schutz, T. Jost, and H. Hugli, "Multi-feature matching algorithm for free-form 3D surface registration," Proc. Fourteenth International Conference on Pattern Recognition, IEEE, 1998, pp. 982-984.

[16] G. C. Sharp, S. W. Lee, and D. K. Wehe, "ICP registration using invariant features," IEEE Trans. Pattern Analysis and Machine Intelligence, vol. 24, pp. 90-102, Jan 2002. 
[17] B. Jiang, J. Tang, B. Luo, and L. Lin, "Robust Feature Point Matching With Sparse Model," IEEE Trans. Image Processing, vol. 23, pp. 5175-5186, 2014.

[18] N. D. Cahill, J. A. Noble, and D. J. Hawkes, "Demons Algorithms for Fluid and Curvature Registration," Proc. 2009 Ieee International Symposium on Biomedical Imaging: From Nano to Macro, IEEE, 2009, pp. 730-733.

[19] S. Henn, "A full curvature based algorithm for image registration," Journal of Mathematical Imaging and Vision, vol. 24, pp. 195-208, Mar 2006.

[20] L. Lin, X. Liu, and S.-C. Zhu, "Layered Graph Matching with Composite Cluster Sampling," IEEE Trans. Pattern Analysis and Machine Intelligence, vol. 32, pp. 1426-1442, 2010.

[21] A. E. Johnson and M. Hebert, "Using spin images for efficient object recognition in cluttered 3D scenes," IEEE Trans. Pattern Analysis and Machine Intelligence, vol. 21, pp. 433449, 1999.

[22] A. Frome, D. Huber, R. Kolluri, T. Bülow, and J. Malik, "Recognizing objects in range data using regional point descriptors," Proc. Computer Vision-ECCV 2004, Springer Berlin Heidelberg, 2004, pp. 224-237.

[23] C. Wu, B. Clipp, X. Li, J. M. Frahm, and M. Pollefeys, "3D model matching with Viewpoint-Invariant Patches (VIP)," Proc. Computer Vision and Pattern Recognition, IEEE, 2008, pp. 1-8.

[24] P. Scovanner, S. Ali, and M. Shah, "A 3-dimensional sift descriptor and its application to action recognition," Proc. the 15th international conference on Multimedia, Augsburg, Germany, 2007, pp. 357-360.

[25] W.-Y. Lin, L. Liu, Y. Matsushita, L. Kok-Lim, and S. Liu, "Aligning images in the wild," Proc. Computer Vision and Pattern Recognition (CVPR), 2012 IEEE Conference on, 2012, pp. 1-8.

[26] F. Tombari, S. Salti, and L. D. Stefano, "Unique Signatures of Histograms for Local Surface Description," Proc. Computer Vision-ECCV 2010, 2010, pp. 356-369.

[27] L. Lin, P. Luo, X. Chen, and K. Zeng, "Representing and recognizing objects with massive local image patches," Pattern Recognition, vol. 45, pp. 231-240, 2012.

[28] L. Lin, X. Wang, W. Yang, and J.-H. Lai, "Discriminatively trained and-or graph models for object shape detection," Pattern Analysis and Machine Intelligence, IEEE Transactions on, vol. 37, pp. 959-972, 2015.

[29] A. L. Yuille, P. W. Hallinan, and D. S. Cohen, "Feature extraction from faces using deformable templates," International journal of computer vision, vol. 8, pp. 99-111, 1992.

[30] D. Zhang and G. Lu, "Shape-based image retrieval using generic Fourier descriptor," Signal Processing: Image Communication, vol. 17, pp. 825-848, 2002.

[31] A. Yezzi Jr, S. Kichenassamy, A. Kumar, P. Olver, and A. Tannenbaum, "A geometric snake model for segmentation of medical imagery," IEEE Trans. Medical Imaging, vol. 16, pp. 199-209, 1997.

[32] S. Osher and R. P. Fedkiw, Level set methods and dynamic implicit surfaces vol. 153: Springer Verlag, 2003.

[33] S. Belongie, J. Malik, and J. Puzicha, "Shape matching and object recognition using shape contexts," IEEE Trans. Pattern Analysis and Machine Intelligence, vol. 24, pp. 509-522, 2002.

[34] Y. Zheng and D. Doermann, "Robust point matching for nonrigid shapes by preserving local neighborhood structures," IEEE Trans. Pattern Analysis and Machine Intelligence, vol. 28, pp. 643-649, 2006.

[35] N. Paragios, M. Rousson, and V. Ramesh, "Matching distance functions: A shape-to-area variational approach for global-tolocal registration," Proc. Computer Vision - Eccv 2002, Springer Berlin Heidelberg, 2002, pp. 775-789.

[36] J. Ma, J. Zhao, J. Tian, Z. Tu, and A. L. Yuille, "Robust Estimation of Nonrigid Transformation for Point Set
Registration," Proc. Computer Vision and Pattern Recognition (CVPR), 2013 IEEE Conference on, 2013, pp. 2147-2154.

[37] H. Chui and A. Rangarajan, "A new algorithm for non-rigid point matching," Proc. Computer Vision and Pattern Recognition, IEEE, 2000, pp. 44-51 vol.2.

[38] H. Chui and A. Rangarajan, "A new point matching algorithm for non-rigid registration," Computer Vision and Image Understanding, vol. 89, pp. 114-141, 2003.

[39] H. Chui, A. Rangarajan, J. Zhang, and C. M. Leonard, "Unsupervised learning of an Atlas from unlabeled point-sets," IEEE Trans. Pattern Analysis and Machine Intelligence, vol. 26, pp. 160-172, 2004.

[40] W. Lian and L. Zhang, "Point Matching in the Presence of Outliers in Both Point Sets: A Concave Optimization Approach," Proc. Computer Vision and Pattern Recognition (CVPR), 2014 IEEE Conference on, 2014, pp. 352-359.

[41] W. Lian and L. Zhang, "Robust Point Matching Revisited: A Concave Optimization Approach," Proc. Computer Vision ECCV 2012, 2012, pp. 259-272.

[42] D. Aiger, N. J. Mitra, and D. Cohen-Or, "4-points congruent sets for robust pairwise surface registration," $A C M$ Transactions on Graphics (TOG), vol. 27, pp. 85:1-85:10, 2008.

[43] B. Ma and R. Ellis, "Surface-based registration with a particle filter," Medical Image Computing and Computer-Assisted Intervention-MICCAI 2004, pp. 566-573, 2004.

[44] B. Ma and R. Ellis, "Unified point selection and surface-based registration using a particle filter," Medical Image Computing and Computer-Assisted Intervention-MICCAI 2005, pp. 75-82, 2005.

[45] M. H. Moghari and P. Abolmaesumi, "Point-based rigid-body registration using an unscented kalman filter," IEEE Trans. Medical Imaging, vol. 26, pp. 1708-1728, 2007.

[46] R. Sandhu, S. Dambreville, and A. Tannenbaum, "Point Set Registration via Particle Filtering and Stochastic Dynamics," IEEE Trans. Pattern Analysis and Machine Intelligence, vol. 32, pp. 1459-1473, 2010.

[47] R. Sandhu, S. Dambreville, and A. Tannenbaum, "Particle filtering for registration of 2D and 3D point sets with stochastic dynamics," Proc. Computer Vision and Pattern Recognition, IEEE, 2008, pp. 1-8.

[48] J. Goldberger, S. Gordon, and H. Greenspan, "An efficient image similarity measure based on approximations of KLdivergence between two gaussian mixtures," Proc. Computer Vision-ECCV 2003, Springer Berlin Heidelberg, 2003, pp. 487493 vol.1.

[49] S. Ge, G. Fan, and M. Ding, "Non-rigid Point Set Registration with Global-Local Topology Preservation," Proc. Computer Vision and Pattern Recognition Workshops (CVPRW), 2014 IEEE Conference on, 2014, pp. 245-251.

[50] D. M. Berg, O. Cheong, and V. M. Kreveld, Computational geometry: algorithms and applications: Springer-Verlag New York Inc, 2008.

[51] S. Umeyama, "Least-squares estimation of transformation parameters between two point patterns," IEEE Trans. Pattern Analysis and Machine Intelligence, vol. 13, pp. 376-380, 1991.

[52] Aim@Shape. (2007). AIM@SHAPE Shape Repositoryv4.0 Available: http://shapes.aimatshape.net

[53] Stanford. The Stanford 3D Scanning Repository. Available: http://graphics.stanford.edu

[54] C. T. Gomez. (2010). BiomedTown Heart Model Dataset. Available: https://www.biomedtown.org/biomed_town/MSV/ reception/wikis/CardiacModelling

[55] R. W. Sumner and J. Popovi, "Deformation transfer for triangle meshes," Acm Transactions on Graphics, vol. 23, pp. 399-405, 2004. 